\title{
Age-Based Structural Dynamics Between Perceptual Speed and Knowledge in the Berlin Aging Study: Direct Evidence for Ability Dedifferentiation in Old Age
}

\author{
Paolo Ghisletta \\ Max Planck Institute for Human Development
}

\author{
Ulman Lindenberger \\ Saarland University
}

\begin{abstract}
According to 2-component theories of intelligence, negative cross-sectional age gradients in mechanic (broad Gf) and pragmatic (broad Gc) cognitive components reflect the increasing constraining of the former in the expression and integrity of the latter component. The authors examined this widely held but untested assumption by applying a recently proposed dynamic structural modeling technique, the bivariate dual change score model, to longitudinal data from the Berlin Aging Study $(N=516$, age range $=70-103$ years). Mechanics and pragmatics were indexed by perceptual speed and knowledge, respectively. As hypothesized, results indicated that changes in knowledge are dominated by perceptual speed and offered strong support for the notion of "mechanization" of pragmatic abilities in old and very old age.
\end{abstract}

Intellectual development in adulthood and old age is multidimensional and multidirectional (Baltes, 1987, 1997; Baltes, Cornelius, Spiro, Nesselroade, \& Willis, 1980; cf. Horn, 1982; Jones $\&$ Conrad, 1933). Intellectual abilities that primarily assess individual differences in biographically acquired knowledge, such as verbal knowledge, tend to remain stable or increase up to middle and late adulthood. In contrast, intellectual abilities presumably related to the speed, accuracy, and coordination of processing, such as perceptual speed, show monotonic decline during adulthood. This difference between aging-resilient and aging-sensitive abilities is at the heart of two-component theories of intelligence (TTIs; for a review, see Lindenberger, 2001). Typical examples include the distinction of Tetens (1777) between absolute and

Paolo Ghisletta, Max Planck Institute for Human Development, Berlin, Germany; Ulman Lindenberger, School of Psychology, Saarland University, Saarbrücken, Germany.

Paolo Ghisletta is now at the Center for Interdisciplinary Gerontology and the Faculty of Psychology and Educational Sciences, University of Geneva, Geneva, Switzerland, and at the Center for Life Course and Life Style Studies, University of Lausanne, Lausanne, Switzerland, and University of Geneva, Geneva, Switzerland. Ulman Lindenberger is now at the Max Planck Institute for Human Development, Berlin, Germany.

Portions of these analyses were previously presented at the meeting of the International Society for the Study of Behavioral Development, Beijing, China, July 2000. Preparation of this article was supported by a grant from the Deutsche Forschungsgemeinschaft to Ulman Lindenberger. We thank Paul B. Baltes, John J. McArdle, and Fumiaki Hamagami for productive discussions and/or programming assistance.

Correspondence concerning this article should be addressed to Paolo Ghisletta, Center for Interdisciplinary Gerontology, University of Geneva, Route de Mon Idée 59, 1226 Thônex, Geneva, Switzerland, or to Ulman Lindenberger, Center of Lifespan Psychology, Max Planck Institute for Human Development, Lentzeallée 94, 14195 Dahlem-Berlin, Germany. E-mail: paolo.ghisletta@cig.unige.ch or lindenberger@ mpib-berlin.mpg.de relative capabilities (see Lindenberger \& Baltes, 1999), the Gf/Gc theory of Cattell (1971) and Horn (1982), and the distinction between the mechanics and pragmatics of cognition proposed by Baltes (1987; Baltes, Lindenberger, \& Staudinger, 1998) in adult life. Generally, TTIs make four central assumptions: (a) Cognitive development reflects the operation and interaction of two components, one biological and the other cultural; (b) during development, the biological component is invested into various cultural domains, leading to the acquisition of culturally defined and transmitted bodies of knowledge (cf. investment theory; Cattell, 1971); (c) the biological component declines after maturity; and (d) the cultural component increases during childhood, adolescence, and adulthood as long as knowledge maintenance and knowledge acquisition outweigh age-based losses in biological potential. All four assumptions have received ample empirical support (for a summary, see Baltes et al., 1998).

The purpose of this article is to model the relation between a marker of broad fluid abilities and one of broad crystallized abilities (Horn, 1989), heretofore referred to as mechanics and pragmatics of intelligence, respectively, in old and very old age. Specifically, we test the hypothesis that old-age decline in pragmatic abilities, if and when it occurs, is driven by negative ageassociated changes in mechanics. According to our impression, this proposition has been silently accepted as a further assumption of TTIs. However, to our knowledge, no attempts have been made so far to test directly the tenability of this assumption (but see McArdle, Hamagami, Meredith, \& Bradway, 2000).

Despite the absence of direct empirical tests, correlational investigations of old-age intelligence are generally consistent with the notion that the mechanics of cognition increasingly dominate age changes as well as individual differences in nominally pragmatic intellectual abilities. Most of the relevant findings in this regard can be subsumed under the heading of dedifferentiation (see also Baltes et al., 1980; Deary et al., 1996; Deary \& Pagliari, 1991; Reinert, Baltes, \& Schmidt, 1966; Schaie, 1962; Schaie, Maitland, 
Willis, \& Intieri, 1998). At the level of interindividual differences, three forms of dedifferentiation can be set apart (Baltes \& Lindenberger, 1997; Lindenberger \& Baltes, 1994, 1997). First, the divergence between mechanic and pragmatic intellectual abilities in old age, with performance decrements for mechanics and stability or increases for pragmatics, is consistent with convergence of age gradients in the sense that both pragmatic and mechanic abilities are negatively related to age (directionality dedifferentiation). Second, correlations among intellectual abilities in old and very old age are higher and more homogeneous than during earlier periods of the adult life span (covariance dedifferentiation within the intellectual-ability domain). Third, the correlational link between intellectual abilities and basic indicators of sensory and sensorimotor functioning is much stronger than at earlier periods of adulthood (covariance dedifferentiation across intellectual, sensory, and sensorimotor domains). Taken together, generalized cross-sectional decrements in level of functioning coupled with increments in covariance seem to indicate an increasing dominance of biological constraints with advancing age.

The aim of this study was not to examine the nature of these constraints at the level of specific cognitive operations or brainrelated mechanisms (e.g., Cabeza, 2001; Li \& Lindenberger, 1999; Raz, 2000; Rubin, 1999) but to test their age-based structural dynamics in the intellectual domain. Our analyses were based on longitudinal and cross-sectional data from the Berlin Aging Study (BASE; Baltes \& Mayer, 1999), as described subsequently. In the BASE, four intellectual abilities were assessed longitudinally: perceptual speed, memory (i.e., episodic memory), fluency, and knowledge (i.e., semantic memory). From these four, perceptual speed and knowledge were selected for analysis in the present study. This decision was guided by two considerations. First, earlier analyses of the BASE data set have shown that perceptual speed and knowledge are the two most divergent intellectual abilities, in terms of both cross-sectional age relations and correlates (Lindenberger \& Baltes, 1997). Specifically, in the initial cross-sectional sample of 516 adults 70 to 103 years of age, perceptual speed had the most negative $(r=-.59)$ and knowledge the least negative $(r=-.42)$ age relations. Also, perceptual speed had significantly higher relations to a factor representing sensory and sensorimotor functioning than did knowledge, whereas knowledge had significantly higher relations to a factor representing differences in years of education, social class, occupational prestige, and income than did perceptual speed. Second, a wealth of data strongly suggests that perceptual speed is an excellent marker of cognitive decline during adulthood and old age (Hertzog, 1989; Salthouse, 1991; Salthouse, Hambrick, Lukas, \& Dell, 1996; Verhaeghen \& Salthouse, 1997). This observation holds true regardless of whether measures of perceptual speed are seen as causally proximal or distal indicators of brain aging.

\section{Methodological Tools for Testing Dynamic Hypotheses About Life Span Development}

The central prediction of this study is dynamic in the sense that one variable is hypothesized to influence changes in another over time (Boker \& Graham, 1998; McArdle \& Bell, 2000; McArdle et al., 2000; Nesselroade \& Ghisletta, 2003). Recently, McArdle and Hamagami (Hamagami \& McArdle, 2001; Hamagami, McArdle, \& Cohen, 2000; McArdle, 2001; McArdle et al., 2000) developed the dual-change score model (DCSM) to test dynamic predictions of this sort. The DCSM relates classical repeated measures models and discrete linear difference equations. It can also be considered to be an extension of a larger class of models that have been introduced into the literature under different names, depending on statistical tradition, such as latent growth curve models (LGMs), multilevel models, random coefficient models, and mixed-effect models (for a comparative and integrative account of these traditions, see McArdle \& Hamagami, 1996). For simplicity, this variety of traditions is heretofore subsumed under the heading of LGMs.

Generally, LGMs postulate a common time-based trajectory for a group and represent the trajectories of individuals as deviations from the group trajectory. The shape of the trajectory can be specified beforehand on theoretical grounds, or it can be estimated empirically. LGMs also accommodate unbalanced and incomplete data, because modeling can be based on both raw data and firstand second-order moments. This implies that individual longitudinal trajectories need not extend over the entire time period considered, in line with earlier versions of combined longitudinal and cross-sectional, or convergence, analysis (Bell, 1953, 1954). The longer the individual trajectories (e.g., the higher the number of individual repeated measures), the more efficient the estimation procedure (i.e., the smaller the parameter estimates' standard errors; cf. McArdle, 1994). Multivariate extensions of LGMs allow one to investigate the degree to which interindividual differences in level and in amounts of change are correlated across two or more variables (Ghisletta \& Lindenberger, in press; Lindenberger \& Ghisletta, in press; MacCallum, Kim, Malarkey, \& KiecoltGlaser, 1997; Raudenbush \& Chan, 1992; Stoolmiller, 1994). By definition, however, standard LGMs assume that population parameters are constant over time. For instance, the correlation between change in variable $A$ and change in variable $B$ is assumed to be constant across all time points. Moreover, such correlations do not necessarily reflect dynamic processes of the kind examined here. Therefore, standard LGMs do not allow for tests of directionality of influence but, at most, for the examination of static structures of interindividual differences among variables' level and change factor scores. In this sense, we cannot derive which variable, if any, is of major influence within the system considered.

In contrast, recent extensions of LGMs such as the DCSM allow researchers to specify and test dynamic (e.g., lead-lag) relations among two or more variables (cf. McArdle et al., 2000; for a related two-occasion method, see McArdle \& Nesselroade, 1994; cf. Hertzog, in press; Hultsch, Hertzog, Dixon, \& Small, 1998). Within the DCSM, the direct effect of one variable on the change of another variable can be examined empirically, because the change in one variable is defined as a time-based function of both itself and the other variable. Applications include the small leading indicator role that the Peabody Individual Achievement Test reading composite exerted on the Behavior Problems Inventory composite (indicative of antisocial behavior) in children 6 to 8 years of age, observed every 2 years for up to four times, in the National Longitudinal Study of Youth (McArdle \& Hamagami, 2001); the potential leading effect of lateral ventricular size on a composite score of episodic memory revealed in the Massachusetts General Hospital Aging Study (McArdle et al., 2003); the leading effect of a nonverbal composite score on a verbal composite score from the Wechsler Intelligence Scale for Children in a sample of first-grade 
children assessed three times (McArdle, 2001); and the examination of several hypotheses of the theory of fluid and crystallized intelligence by studying the dynamic relationships among composite scores of broad knowledge, spatial reasoning, perceptual speed, and immediate memory from the Wechsler Adult Intelligence Scale (WAIS; McArdle et al., 2000).

With such models, the following questions can be formalized and made amenable to sound statistical testing: (a) Does variable $A$ (or $B$ ) influence the subsequent change of variable $A$ (or $B$ )? (b) Does variable $A$ (or $B$ ) influence the subsequent change of variable $B$ (or $A$ )? and (c) Does the effect of variable $A$ on the subsequent change of variable $B$ differ from the effect of variable $B$ on the subsequent change of variable $A$ ? In this manner, the multivariate DCSM allows for a better understanding of dynamic structures than does the multivariate study of change achieved by twooccasion latent difference score models (McArdle \& Nesselroade, 1994) or traditional multivariate LGMs. Specifically, in the present theoretical context, DCSMs can be used to test formally the proposition that mechanics are the driving force of intellectualability dedifferentiation in old and very old age, with respect to both age gradients and degree of covariance.

\section{Method}

\section{Participants}

The BASE is a multidisciplinary investigation of old and very old residents of former West Berlin (Baltes \& Mayer, 1999; Baltes, Mayer, Helmchen, \& Steinhagen-Thiessen, 1993). The parent sample consisted initially of 516 participants and originated from a random draw of addresses from the city registry (for details, see Lindenberger et al., 1999). This initial sample was stratified across sex and age, such that $2(\operatorname{sex}) \times 6$ (age) groups $(70-74,75-79,80-84,85-89,90-94$, and 95 years and older) were sampled, with 43 participants in each group. The main rationale for this design was to optimize power for detecting main effects and interactions regarding age and gender (cf. McClelland \& Judd, 1993). The present analyses were based on the first three major measurement occasions. Sample size decreased from 516 participants at Time 1 (1990-1993) to 206 at Time 2 (1995-1996) and 132 at Time 3 (1997-1998). Therefore, the models used must account for patterns of incomplete data due to dropout.

The time span modeled in the present study (i.e., 34 years) was large relative to the mean longitudinal observation period (1.98 years). Therefore, the present analyses were not well suited to assess interindividual differences in intraindividual change; instead, the focus was on dynamic age changes at the population level. Hence, in the present study, the statistical parameters operationalizing evidence in favor of or against the dedifferentiation hypothesis were general, population-level parameters and not interindividual-difference parameters (e.g., in multilevel terminology, they were fixed rather than random effects). Specifically, the limited within-person longitudinal information did not allow us to estimate individual variations (i.e., random effects) around the dynamic parameters expressing the dedifferentiation hypothesis at the population level. Nevertheless, within-person longitudinal change information was necessary for proper identification of population dynamics (see the Statistical Models section for details).

\section{Violation of Missing-at-Random Assumption}

Attrition in the BASE tended to be nonrandom, or selective (Lindenberger et al., 1999; Lindenberger, Singer, \& Baltes, 2002; Singer, Verhaeghen, Ghisletta, Lindenberger, \& Baltes, 2003). To assess the relevance of this issue for the present study, we examined longitudinal selectivity of perceptual speed and knowledge following procedures described by Lindenberger et al. (2002). Specifically, selectivity was expressed in an effect-size metric indicating the degree to which the individuals with data on all three occasions $(n=132)$ differed from the parent sample $(N=516)$ from which they originated. Two additive components were distinguished, one related to biological mortality and the other to nonparticipation of survivors (i.e., mortality-associated vs. experimental selectivity; for computation, see Lindenberger et al., 2002). Total selectivity for perceptual speed and knowledge measured at the first occasion amounted to 0.774 standard deviation units for perceptual speed (mortality-associated component $=0.480$, experimental component $=0.294)$ and 0.494 standard deviation units for knowledge (mortality-associated component $=0.276$, experimental component $=0.218$ ), where the standard deviation refers to that of the parent sample of 516 people. These results indicate that attrition in the present study was nonrandom, or selective, for both experimental and mortality-associated reasons. Implications of nonrandom attrition for the interpretability of the results obtained in this study are considered in the Discussion section.

\section{Cognitive Abilities Examined}

The longitudinal cognitive battery of the BASE consisted of four cognitive domains measured with two variables each, or a total of eight variables (Lindenberger, Mayr, \& Kliegl, 1993). The present study was restricted to perceptual speed and knowledge. The Digit Letter and Identical Pictures tests were used to assess perceptual speed, whereas the Vocabulary and Spot-a-Word tests were used to assess knowledge.

The Digit Letter test resembles the Symbol Substitution test of the WAIS (Wechsler, 1955). Participants were shown, throughout the entire duration of the test ( $3 \mathrm{~min})$, a template with nine digit-letter pairings. Participants were then shown up to 21 series of six digits; they had to pair each digit with its correct letter according to the template, as quickly as possible. The Identical Pictures test was a computerized version of the homonymous test of the Educational Testing Service (Ekstrom, French, Harman, \& Dermen, 1976). A target and five response alternative figures were shown on the computer screen. Participants were asked to touch the correct response figure, identical to the target, as quickly as possible, within $80 \mathrm{~s}$. The Vocabulary test consisted of 20 words, shown on the computer screen, that participants were asked to define without time constraints. The definitions were then coded (as incorrect, partially correct, or correct) by two independent raters according to instructions provided by Wechsler (1982). Finally, the Spot-a-Word test consisted of 20 series of one word and four pronounceable nonwords shown on the computer screen. Participants were asked to touch the word.

A unit-weighted linear composite of the Digit Letter and Identical Pictures test scores (reexpressed to have analogous scaling properties at Time 1) was computed to obtain a perceptual speed score. Likewise, the Vocabulary and Spot-a-Word test scores were combined to obtain a knowledge score. Both composite scores were rescaled to have a mean of 50 and a standard deviation of 10 in the parent sample at Time 1. This transformation did not alter the psychometric properties of the scores, retained longitudinal changes in mean and variance, and allowed for reasonable, meaningful comparisons and ease of interpretability.

Table 1 presents the summary longitudinal and cross-sectional, or convergence, statistics for the perceptual speed and knowledge composite scores at each age from 70 to 104 years (the oldest person of 103 years in the cross-sectional sample aged 1 year in the longitudinal design). The mean and standard deviation of all observations for all individuals are presented by age of assessment, independent of the measurement pattern of the participants. The trajectory examined spanned 34 years, equivalent to 35 possible ages, from the first measurement of the youngest participant to the last measurement of the oldest participant. As can be expected, neither composite score distribution was rectangular, as would be the case if 
Table 1

Descriptive Statistics for Perceptual Speed and Knowledge Composite Scores Measured in the Berlin Aging Study as a Function of Age of Assessment

\begin{tabular}{|c|c|c|c|c|c|}
\hline Age & $n$ & $M_{S}$ & $V_{S}$ & $M_{K}$ & $V_{K}$ \\
\hline 70 & 7 & 59.78 & 83.10 & 56.85 & 53.20 \\
\hline 71 & 18 & 59.43 & 40.43 & 54.55 & 46.44 \\
\hline 72 & 13 & 58.52 & 29.72 & 56.72 & 53.75 \\
\hline 73 & 22 & 59.65 & 49.18 & 55.26 & 66.96 \\
\hline 74 & 22 & 57.84 & 73.28 & 53.76 & 72.00 \\
\hline 75 & 32 & 57.66 & 47.42 & 53.80 & 62.46 \\
\hline 76 & 30 & 56.25 & 56.97 & 56.28 & 44.16 \\
\hline 77 & 31 & 57.97 & 65.22 & 56.67 & 76.99 \\
\hline 78 & 39 & 56.55 & 40.40 & 51.32 & 68.95 \\
\hline 79 & 38 & 57.35 & 50.13 & 54.98 & 79.65 \\
\hline 80 & 52 & 56.08 & 71.77 & 51.49 & 84.94 \\
\hline 81 & 32 & 53.42 & 11.17 & 53.67 & 94.66 \\
\hline 82 & 39 & 56.53 & 36.74 & 53.72 & 67.53 \\
\hline 83 & 37 & 51.58 & 90.50 & 48.53 & 104.51 \\
\hline 84 & 28 & 55.20 & 28.30 & 52.54 & 69.74 \\
\hline 85 & 43 & 52.86 & 103.87 & 52.81 & 99.08 \\
\hline 86 & 36 & 52.24 & 106.74 & 51.62 & 105.47 \\
\hline 87 & 33 & 48.35 & 108.85 & 48.22 & 88.07 \\
\hline 88 & 33 & 51.86 & 82.52 & 52.80 & 70.24 \\
\hline 89 & 27 & 48.60 & 90.01 & 49.66 & 68.56 \\
\hline 90 & 19 & 46.38 & 76.98 & 52.53 & 60.78 \\
\hline 91 & 27 & 47.02 & 72.02 & 50.36 & 75.80 \\
\hline 92 & 30 & 44.75 & 64.10 & 46.73 & 85.30 \\
\hline 93 & 21 & 46.09 & 74.44 & 49.28 & 76.94 \\
\hline 94 & 16 & 41.27 & 74.53 & 44.75 & 89.30 \\
\hline 95 & 27 & 44.46 & 47.85 & 48.30 & 84.91 \\
\hline 96 & 26 & 43.68 & 55.79 & 45.40 & 117.55 \\
\hline 97 & 23 & 41.77 & 69.24 & 46.92 & 12.43 \\
\hline 98 & 12 & 45.42 & 17.60 & 48.36 & 36.80 \\
\hline 99 & 12 & 40.35 & 24.64 & 45.82 & 42.24 \\
\hline 100 & 11 & 45.29 & 80.18 & 44.26 & 68.65 \\
\hline 101 & 10 & 42.09 & 73.55 & 41.53 & 102.26 \\
\hline 102 & 2 & 36.84 & 38.25 & 40.00 & 122.88 \\
\hline 103 & 3 & 54.16 & 12.54 & 56.43 & 27.00 \\
\hline 104 & 1 & 46.33 & 0.00 & 58.51 & 0.00 \\
\hline
\end{tabular}

Note. Age in years is participants' age of assessment; $M_{S}$ is the mean of the perceptual speed composite score; $V_{S}$ is the variance of the perceptual speed composite score; $M_{K}$ is the mean of the knowledge composite score; $V_{K}$ is the variance of the knowledge composite score.

observations were restricted to the age-stratified first-occasion sample. At high ages, observations are less frequent because of age-correlated attrition. At very low ages, observations are less frequent because of the age-stratified design. Figures 1 and 2 plot the individual longitudinal age trajectories for perceptual speed and knowledge, respectively. In both figures, lines unify longitudinal data points of the same participants, and circles represent the scores of participants assessed only once. The reduced likelihood of repeated individual measurements is represented by the higher relative density of circles at older ages.

\section{Statistical Models}

Figure 3 represents a graphical depiction of an LGM in one form of structural equation modeling (McArdle \& McDonald, 1984; McArdle \& Nesselroade, 2002), adapted to the BASE longitudinal design. In this graphical notation, squares represent manifest variables, circles represent latent variables, and the triangle represents a constant value of unity, inserted to represent intercepts (following Boker, McArdle, \& Neale, 2002;
McArdle \& Boker, 1990). Moreover, one-headed, asymmetrical arrows represent directional regression (deterministic) coefficients, whereas twoheaded, symmetrical arrows represent variances or covariances (stochastic coefficients). Circles inside squares represent variables that might be manifest (e.g., measured) for some participants while being latent (e.g., unmeasured) for others. Squares represent the actual measurement pattern of a given individual. For instance, the data vector of a person measured twice, at 82 and 86 years of age, would be represented by a circle at each age from 70 to 104 years except for 82 and 86 years and by a square at those two ages of actual assessment.

This graphical notation allows for an efficient representation of a large number of individual measurement schedules as well as various patterns of data incompleteness. This notation accommodates BASE participants who were measured on all three occasions as well as those assessed only twice or once. For example, the same diagram can be used to represent a participant measured on all three occasions at 72,76 , and 78 years of age; another measured twice at 93 and 97 years of age; and a participant assessed only once at 101 years of age. Here we are interested in the process of dedifferentiation of cognitive abilities in old age, unfolding from 70 to 104 years of age. Hence, study of cognitive change as a function of time in the study is not adequate in this application. The data, their representation (i.e., Figures 1 and 2), and the analyses are thus organized such that individual segments (averaging 1.98 years in length) are juxtaposed to form a population curve spanning more than 34 years (as in, e.g., Ghisletta \& McArdle, 2001).

The self-directed two-headed arrows denoted $R$ for each circle inside a square symbolize residual variance. It is assumed here that residual variance does not change or correlate over time. Thus, the residual variance matrix follows a simple diagonal structure, with identical values at all ages. The rest of the variance of each measurement is modeled by enforcing a particular change process by means of latent level and change variables. The circle denoted $L$ represents the level score of the participants, and the two-headed arrow about this circle, denoted $V_{L}$, represents its variance (thus, individual differences on level scores). Analogously, the circle denoted $S$ represents the slope (or change) score of the participants, which also allows for individual deviations $\left(V_{S}\right)$. The two-headed arrow denoted $C_{L S}$, from the level to the slope factor, represents the covariance between the latent level and slope variables. The regression paths $M_{L}$ and $M_{S}$ from the triangle denoted 1 to the level and slope scores represent the estimation of the means of the level and slope factors (i.e., the average cross product between a constant of value 1 and the level and slope scores, respectively).

The level factor postulates no change, because the magnitude of its relationship (i.e., loadings) to the time series (represented by subsequent measurements) is constant. Hence, the level factor relates with constant magnitude across all measurements of the time series. This is expressed in Figure 3 by setting all loadings of the level factor to equal magnitude (e.g., 1). The shape of the curve examined is defined by the loadings of the slope factor. In Figure 3, the slope loadings are linear with respect to the time intervals. For each successive increment in time units, the corresponding loading augments by a constant amount (e.g., 1). In this application, the change process is modeled as a function of chronological age (i.e., time is chronological age). Therefore, each 1-year increment in age corresponds to an increase in the respective loading magnitude of one. The shape of longitudinal change is thus linear, and the figure represents a linear LGM. This graphical notation explicitly symbolizes all parameters of the model and allows for direct calculation of expectations by applying the tracing rules first introduced by Wright $(1918,1920)$ to the average cross products (see Boker et al., 2002; McArdle \& McDonald, 1984).

Figure 4 depicts an alternative model that, under certain assumptions, is identical to the linear LGM presented in Figure 3. McArdle and Hamagami developed this model and called it the "dual-change score model" (Hamagami \& McArdle, 2001; Hamagami et al., 2000; McArdle, 2001; McArdle \& Hamagami, 1999; McArdle et al., 2000). Historically, this model can be seen as a combination of LGMs and other classical repeated 


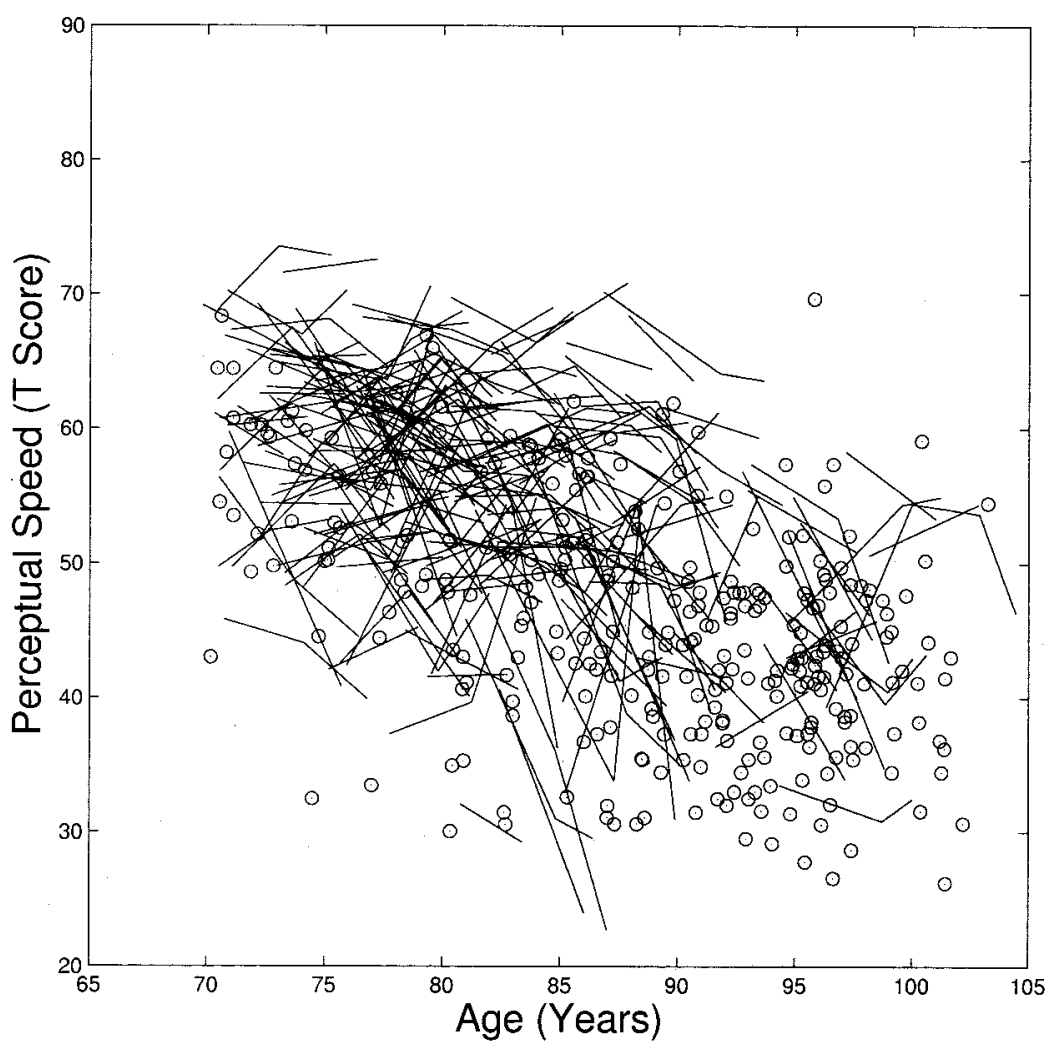

Figure 1. Plot of individual longitudinal trajectories on perceptual speed composite score ( $T$ score, $M=50$, $S D=10$, at Occasion 1) by chronological age (years). Lines unify longitudinal data of the same participants, and circles represent measurements of participants assessed only once.

measures models (such as the use of latent difference scores in twooccasion data introduced by McArdle \& Nesselroade, 1994) with discrete linear difference equations (Arminger, 1986). The model formalizes the difference score between true measurements at adjacent time points. The circles denoted $\Delta Y_{t}$, where $t$ is chronological age ranging from 70 to 104 years, represent the true, or latent, difference score between $Y$ at time $t-$ 1 and $Y$ at time $t$. This can be seen by the fact that at any time point $t, Y_{t}$ is the sum of $Y_{t-1}$ and $\Delta Y_{t}$ (both additive elements are indicated by the regression paths moving from them to the variable $Y_{t}$ with fixed weight of 1). That is, the true score at any time point is the sum of the previous true score and the true difference (i.e., change score) to the next time point. The traditional interpretation of difference scores would be limited by this structure, in that it usually does not allow the previous measurement $Y_{t-1}$ to be correlated to the difference $\Delta Y_{t}$. The DCSM, instead, postulates a coefficient $\beta$ representing the effect of $Y_{t-1}$ on $\Delta Y_{t}$, that is, the effect (or the proportion) of $Y$ at any time point on the magnitude of subsequent change during the specified unit of time (1 year in the present case). The latent score $L$ represents the value of $Y$ at the beginning of the time series (at $t=0$, or 70 years of age in this application). This initial intercept allows for individual differences (as symbolized by its variance $V_{L}$ ). The latent score $S$ represents the change curve of the consecutive latent differences, and the loadings $\alpha$ of this slope factor determine the shape of the curve. The factor $S$ thus models the shape of the longitudinal change of the consecutive difference scores and allows for individual differences in change $\left(V_{S}\right)$.

By constraining the values of $\alpha$ and $\beta$, several classical hypotheses of change can be formalized. For instance, when both $\alpha$ and $\beta$ equal 0 , the latent difference scores are not defined, meaning that the difference between any two adjacent measurements is zero. As such, the DCSM with $\alpha$ $=\beta=0$ represents a no change score model, postulating a constant longitudinal score equal to the initial level score for the whole time series (that is, the only one-headed arrow going into $Y$ at any time point originates from the latent level $L$ score). This model estimates three parameters: (a) mean initial level score $\left(M_{L}\right)$, (b) variance around the mean level score $\left(V_{L}\right)$, and (c) residual variance $(R)$. This is a traditional LGM without any slope factor.

By setting $\alpha$ to 0 and estimating $\beta$, we obtain a proportional change score model. This model formalizes the hypothesis that the difference between any two adjacent scores is directly proportional to the previous score, through the parameter $\beta$. The previous score is thus the only effect on the difference score, because this model postulates no slope factor. A proportional change score model estimates four parameters: (a) mean initial level score $\left(M_{L}\right)$, (b) variance about the mean level score $\left(V_{L}\right)$, (c) residual variance $(R)$, and (d) the proportionality parameter $(\beta)$. This is equivalent to a traditional reliable Markov simplex model (Guttman, 1954; McArdle \& Aber, 1990; Wold, 1949), wherein the autoregressive parameter defining the magnitude of the effect of the preceding value $Y_{t-1}$ on $Y_{t}$ is equivalent to $1+\beta$. At times, this is interpreted as the stability component of the time series. If $\beta$ differs reliably from 0 , the expected longitudinal change is nonlinear.

When $\alpha=1$ and $\beta=0$, the DCSM is simplified to represent a model in which the latent difference scores are not directly proportional to the previous measurements and do not change across time. As such, the difference between the values of any two adjacent $Y$ scores is a sole direct function of the time-invariant slope $S$. Because the loadings $\alpha$, defining the shape of the change of the $\Delta Y_{t}$ scores, are fixed at one, no change in $\Delta Y_{t}$ is postulated. The change of the difference scores is nonexistent, and thus 


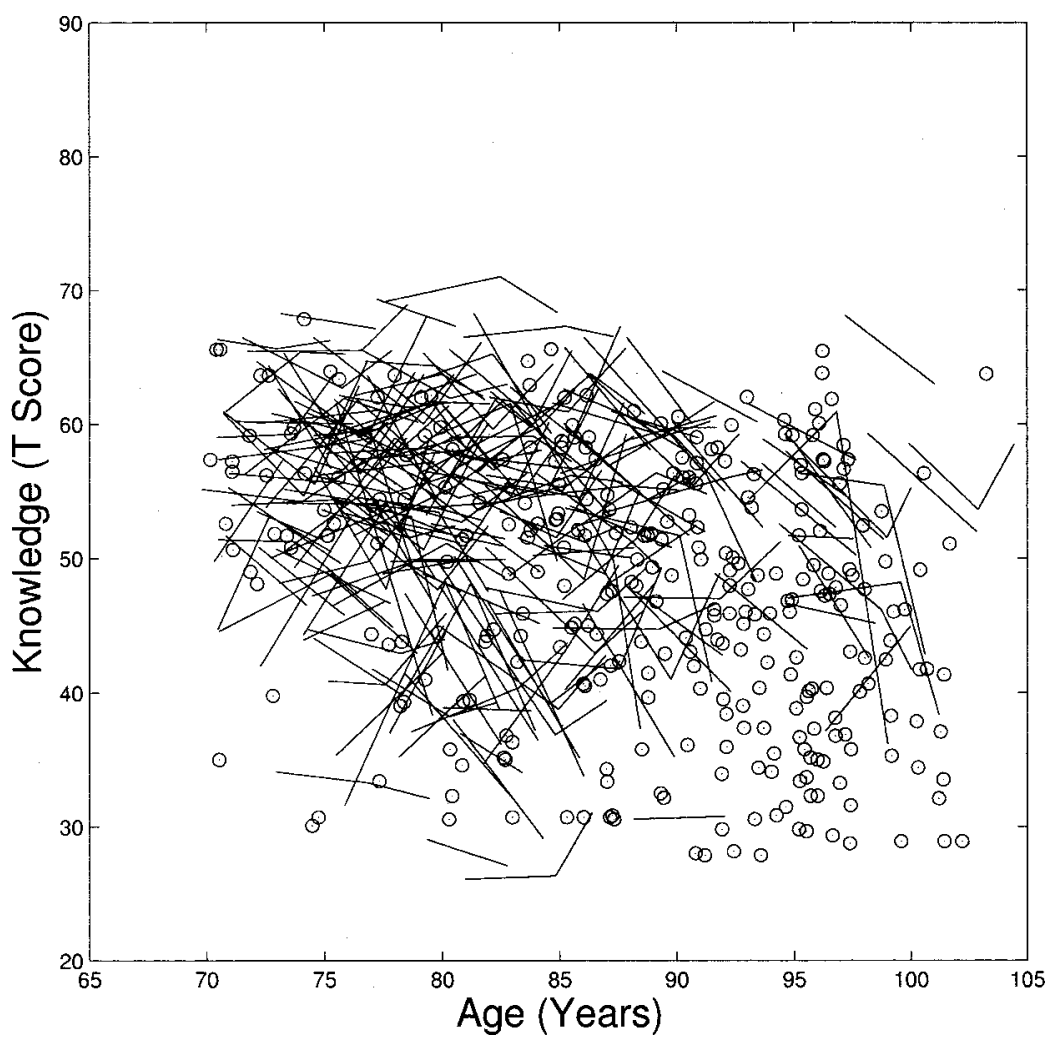

Figure 2. Plot of individual longitudinal trajectories on knowledge composite score ( $T$ score, $M=50, S D=$ 10, at Occasion 1) by chronological age (years). Lines unify longitudinal data of the same participants, and circles represent measurements of participants assessed only once.

the difference scores are constant. That is, between each two consecutive time points $t-1$ and $t$, the effect of the slope score $S$ on the difference scores $\Delta Y_{t}$ is constant. This model is called a constant change score model (CCSM) and is equivalent to the linear LGM of Figure 3. The same six parameters of the linear LGM are estimated, and identical estimates are obtained. The parameters estimated are the mean of the level and slope factors $\left(M_{L}\right.$ and $\left.M_{S}\right)$, the variance of the level and slope factors $\left(V_{L}\right.$ and $\left.V_{S}\right)$, the covariance between the level and slope factors $\left(C_{L S}\right)$, and the residual variance $(R)$. Because this CCSM and a linear LGM are equivalent, the same fit statistics obtain.

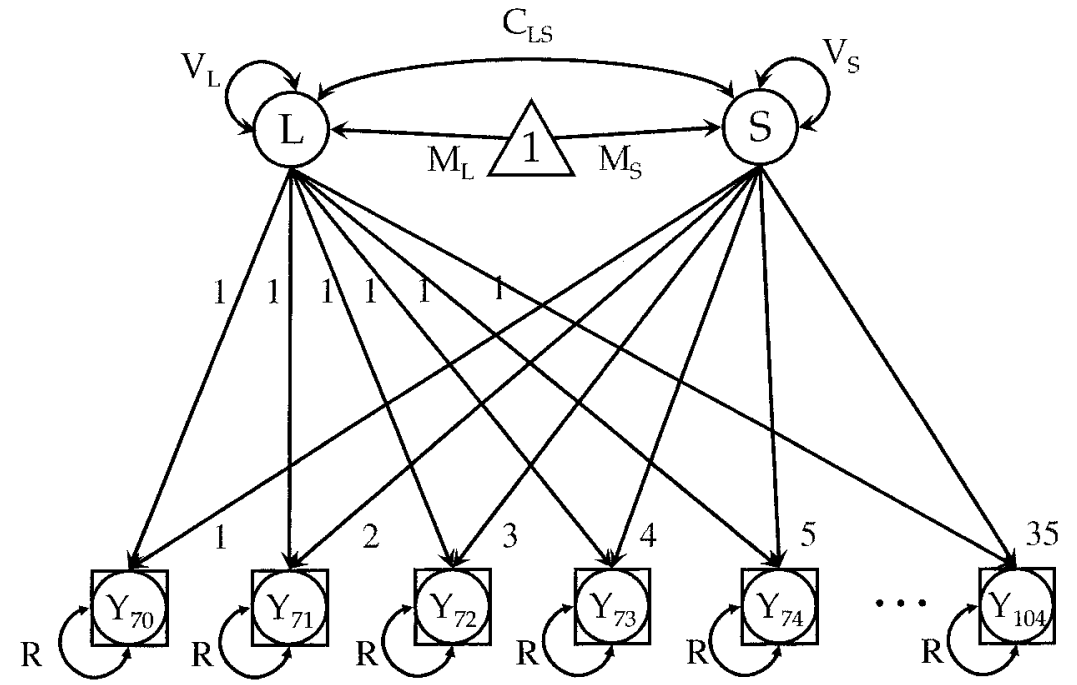

Figure 3. Graphical representation of a latent growth curve model (or constant change score model). 


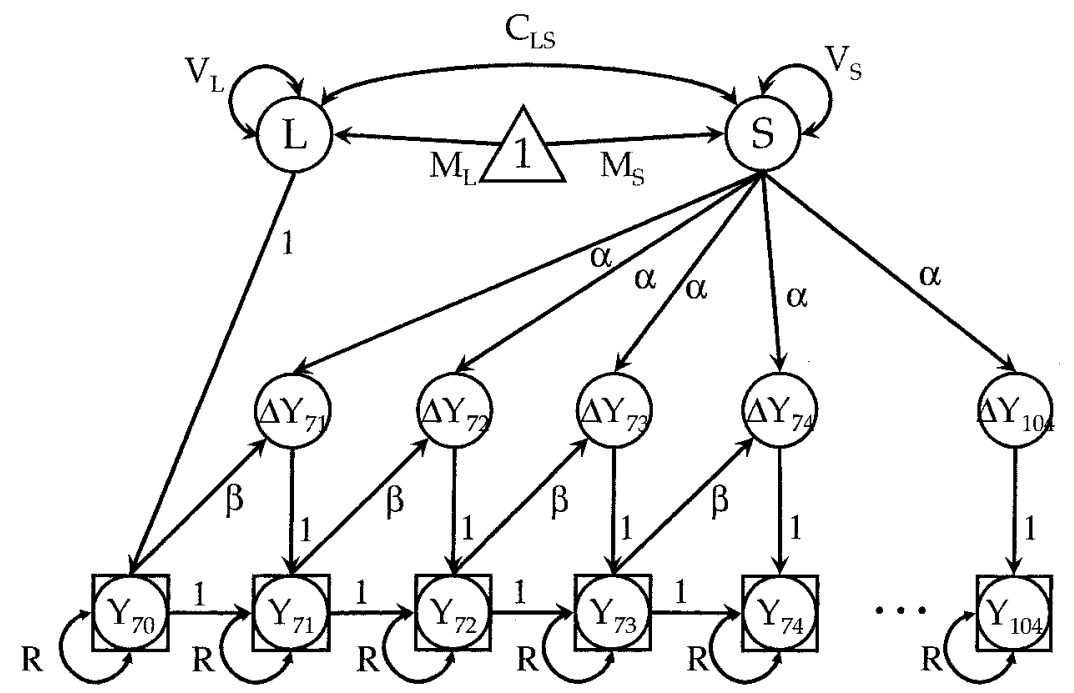

Figure 4. Graphical representation of a univariate dual-change score model.

Finally, the case of $\alpha=1$ and $\beta$ estimated represents the full DCSM. Here one additional parameter is estimated: the value of the autoproportion $\beta$ parameter. This additional parameter defines the $\Delta Y$ score as a function of the slope factor and of the previous $Y$ score. One can thus test the effect of variable $Y$ on the subsequent change in $Y$. The parameter $\beta$ is commonly assumed, for simplicity, to be constant across time, although this is a testable assumption. Moreover, the lag of the auto-proportion effect can be expanded and tested as well. For instance, it might be postulated that $Y_{t}$ does not influence $\Delta Y_{t+1}$ but influences $\Delta Y_{t+2}$, thus implying a 2-year lag instead of a 1-year lag. In the present study, $\beta$ was assumed to be constant over time, and it refers to 1-year effects.

Figure 5 represents a bivariate DCSM (BDCSM). In this figure, for simplification, paths with fixed values of 1 are not labeled. Here two time series are represented, for variable $X$ and for variable $Y$. The repeated measures of the two time series are hypothesized to change according to the individual best specification of a DCSM. Thus, each time series, taken by itself, is described by at most seven parameters (depending on the best-fitting specification of the DCSM). Moreover, the two time series are

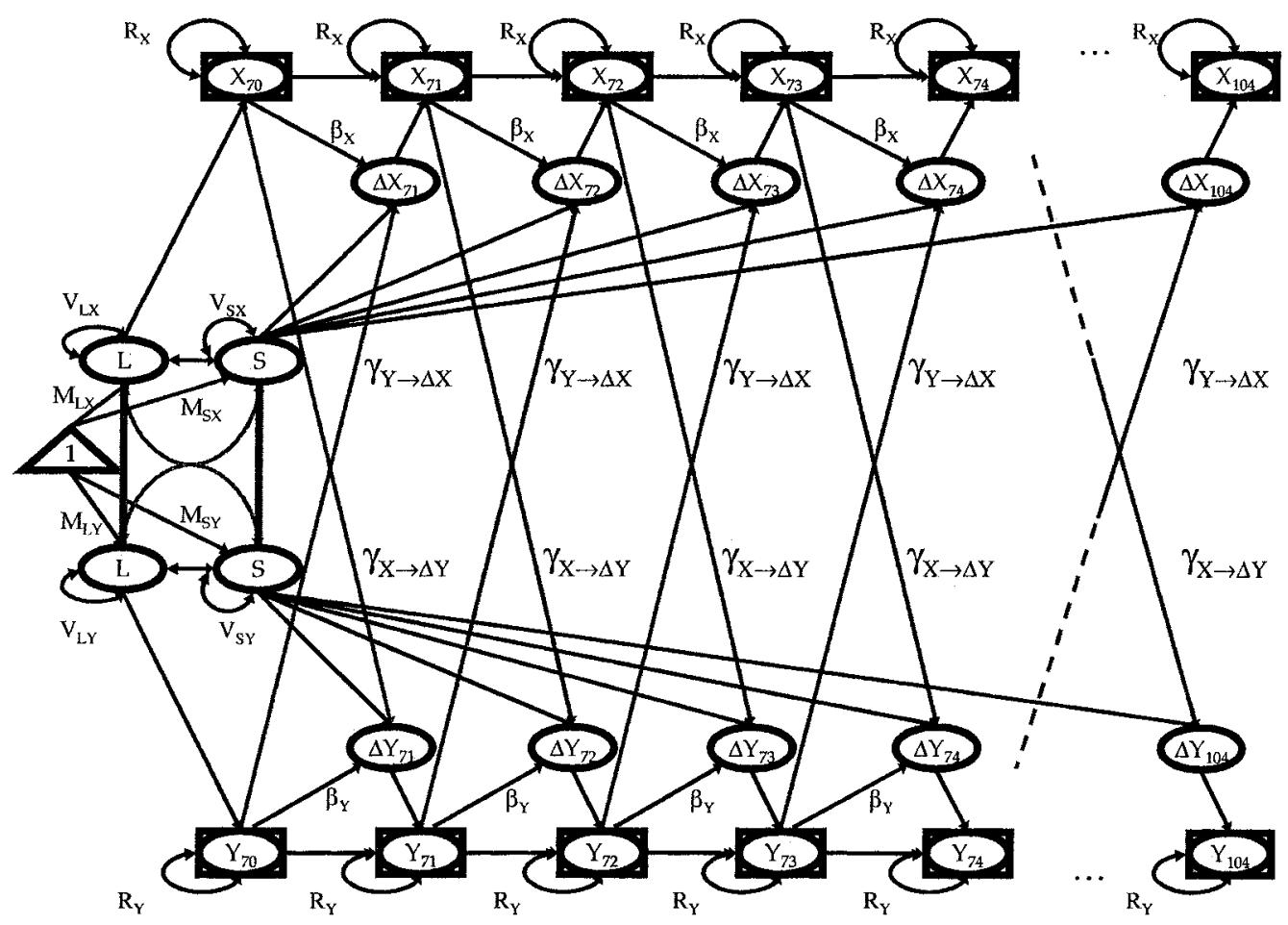

Figure 5. Graphical representation of a bivariate dual-change score model. Unlabeled paths are fixed at 1 . 
linked to allow for correlations between the $X$ and $Y$ variables. Six additional parameters are estimated for this purpose. Four refer to the covariance between the level of $Y$ and the level of $X$, the level of $Y$ and the slope of $X$, the slope of $Y$ and the level of $X$, and the slope of $Y$ and the slope of $X$. Finally, two additional parameters are estimated to allow the two variables to affect each other in a dynamic way. These are denoted $\gamma_{Y \rightarrow \Delta X}$ and $\gamma_{X \rightarrow \Delta Y}$. The $\gamma$ parameters represent, respectively, the effect of $Y$ on the subsequent change in $X$ and the effect of $X$ on the subsequent change in $Y$. As such, the expectations for either of the two time series are a direct function of the variable of one time series, the change in the variable of that time series, and the variable of the other time series.

The traditional LGM captures the constant change feature (i.e., $\alpha=1$ and $\beta=0$ ) of the change process of a variable. The predicted value of a variable at time $t$ is defined by the sum of (a) its predicted value at time $t-$ 1 and (b) the time-independent mean slope. For variable $X$, the resulting equation is

$$
\hat{X}_{t}=\hat{X}_{t-1}+M_{S(X)} .
$$

For variable $Y$, the equation is

$$
\hat{Y}_{t}=\hat{Y}_{t-1}+M_{S(Y)} .
$$

The BDCSM combines the constant change feature (i.e., $\alpha=1$ ) with the proportionality aspect (i.e., $\beta \neq 0$ ) of a change process. The predicted value of a variable at time $t$ is defined by the sum of (a) its predicted value at time $t-1$, (b) the auto-proportion $\beta$ multiplied by its predicted value at time $t-1$, (c) the latent cross-lagged effect $\gamma$ multiplied by the predicted value of the other variable at time $t-1$, and finally (d) the timeindependent mean slope. For variable $X$, this can be represented by the equation

$$
\hat{X}_{t}=\hat{X}_{t-1}+\beta_{X} \cdot \hat{X}_{t-1}+\gamma_{Y \rightarrow \Delta X} \cdot \hat{Y}_{t-1}+\alpha \cdot M_{S(X)},
$$

which simplifies to, assuming $\alpha=1$,

$$
\hat{X}_{t}=\left(1+\beta_{X}\right) \cdot \hat{X}_{t-1}+\gamma_{Y \rightarrow \Delta X} \cdot \hat{Y}_{t-1}+M_{S(X)} .
$$

For variable $Y$, the equation is

$$
\hat{Y}_{t}=\hat{Y}_{t-1}+\beta \cdot \hat{Y}_{t-1}+\gamma_{X \rightarrow \Delta Y} \cdot \hat{X}_{t-1}+\alpha \cdot M_{S(Y)},
$$

which simplifies to, assuming $\alpha=1$,

$$
\hat{Y}_{t}=\left(1+\beta_{Y}\right) \cdot \hat{Y}_{t-1}+\gamma_{X \rightarrow \Delta Y} \cdot \hat{X}_{t-1}+M_{S(Y)} .
$$

By expanding Equation 4 and solving for $\beta$, we obtain

$$
\beta_{X}=\frac{\hat{X}_{t}-\hat{X}_{t-1}-\gamma_{Y \rightarrow \Delta X} \cdot \hat{Y}_{t-1}-M_{S(X)}}{\hat{X}_{t-1}} .
$$

Likewise, Equation 4 can be expanded and solved for $\gamma$ :

$$
\gamma_{Y \rightarrow \Delta X}=\frac{\hat{X}_{t}-\hat{X}_{t-1}-\beta_{X} \cdot \hat{X}_{t-1}-M_{S(X)}}{\hat{Y}_{t-1}} .
$$

Equations 7 and 8 include values measured at time $t$ and $t-1$, and $\hat{X}_{t}-$ $\hat{X}_{t-1}$ represents the latent change score in $X$. Therefore, to estimate $\beta$ and $\gamma$, at least some individual repeated measurements are needed. Indeed, to obtain full identification of the parameters $\beta$ and $\gamma$, longitudinal information is needed. Cross-sectional data do not provide information about correlations over time, which have algebraic expectations for the dynamic parameters of the DCSM. It follows that without longitudinal data there exist no second derivatives and, consequently, no standard errors; thus, there is no unique estimation. Application of the DCSM to cross-sectional data only could provide a solution, and potentially the correct one. However, because of the lack of full model identification, this solution is not rejectable (J. J. McArdle, personal communication, December 2, 2002). As a check, we analyzed purely cross-sectional Time 1 data, and the software was not able to start the needed iterations, even with a wide range of differing starting values (because of the lack of full identification). In our empirical application, estimation of the $\beta$ and $\gamma$ dynamic parameters was possibly only based on the full sample (i.e., cross-sectional and longitudinal) data.

The $\gamma$ parameters allow for testing cross-lagged effects between the two time series at the level of reliable, true latent scores. This information is not captured by the more familiar levels-slopes correlations of multivariate LGMs. If we (a) limit, through parameter constraints, the BDCSM to a no-change model (i.e., $\beta_{X}=\beta_{Y}=0$ and $\alpha_{X}=\alpha_{Y}=0$ ); (b) do not take into account any unique variance of the time series (i.e., $R_{X}=R_{Y}=0$ ); and (c) apply the resulting constrained model to only two time points, we are left with the familiar cross-lagged correlational analysis, with its known limitations. The $\gamma$ parameter then would not predict the asymmetrical effect of the true score of one variable onto the true change score of the other variable. Rather, it would represent the direct effect one variable at time $t-1$ exerts on the other variable at time $t$, without accounting for error and unrelated variance and without separating level information (the variable's score at time $t-1$ ) from change information (the variable's change between times $t-1$ and $t$ ).

As with the auto-proportion parameters $\beta$, one need not assume that the cross-lagged parameters $\gamma$ are constant over the whole time series considered. This, again, is an empirical and testable issue (if data are sufficiently dense to permit empirical identification, for example, if the attrition is minor and if the design is such that participants have been measured more than three times). In this application, we assumed both auto-proportion and cross-lagged parameters to be constant over the entire time span considered. Further details on the DCSM described here, as well as variations and extensions, have been provided by McArdle et al. (2000, 2003), McArdle (2001), McArdle and Hamagami (2001), Hamagami et al. (2000), and Hamagami and McArdle (2001).

Cross-lagged correlations were very popular in the 1980s for addressing questions of reciprocal causal effects. However, as discussed by Rogosa (1980a), there are several problems associated with this analysis. Crosslagged correlations (a) can be the expression of more than one underlying (and often unspecified) causal mechanism, (b) can be very misleading with respect to the true causal model (a large difference between two crosslagged correlations in a two-wave, two-variable design may indicate unequal causal effects as well as the absence of or equal causal effects), (c) rest on very strict and often untenable assumptions for straightforward interpretation (e.g., no change in variance and synchronous correlations over time in the most frequently assumed underlying structural model), and (d) usually do not adjust for measurement error (however, doing so does not necessarily eliminate the remaining limitations; Rogosa, 1980b).

The BDCSM, of course, is no panacea. However, it offers several advantages over cross-lagged correlations. The BDCSM (a) defines explicitly the underlying structural model, which does allow for several change functions frequently adopted in the literature; (b) allows for clearer interpretation of the focus parameters; (c) rests on fewer, more realistic assumptions (we provide more detail in the Discussion section); and (d) accounts for unreliability. Moreover, the BDCSM, as is the case with any structural equation model, can be compared statistically with different theoretical models (e.g., with different change functions specified).

\section{Results}

First we present the results of the univariate analyses. Both the perceptual speed and knowledge composite scores are analyzed separately with the no change, constant change, proportional change, and dual-change models. Then the BDCSM is presented with the latent cross-lagged regression relationships. The central prediction of the present study was tested through specification of equality constraints for the two cross-lagged $(\gamma)$ regression 
weights. Relative to a model with freely estimated cross-lagged regression weights, we expect these equality constraints to be associated with decrements in model fit, because the effect of level-speed on change-knowledge is hypothesized to be greater than the effect of level-knowledge on change-speed.

To familiarize the reader with the kinds of inferences we are trying to reach, we first present traditional time-lagged withinvariable and between-variables correlations. We are aware that the interpretation of such correlations is open to methodological criticism, especially if the reliabilities of the two measures are unequal (Rogosa, 1980b). The main purpose of these preliminary analyses was to facilitate the conceptual transition to the final BDCSM.

\section{Temporal Correlations Over Occasion Lags}

Before formally testing the question "Is the effect of perceptual speed on later changes in knowledge stronger than the effect of knowledge on later changes in perceptual speed?" we examine this issue with cross-lagged correlations, a rather rudimentary analysis that does not account, among other things, for reliability issues. Here we correlate Time 2, Time 1, and again Time 1 scores of the perceptual speed composite with, in order, Time 3, Time 2, and again Time 3 perceptual speed and knowledge scores. Analogously, we correlated earlier scores of the knowledge composite with later scores of knowledge and perceptual speed. This allowed us a first, tentative look at the question "Does perceptual speed correlate more highly with later knowledge than vice versa?"

Table 2 presents these correlations computed across occasions of measurement. Because the occasions of testing were not equidistant in time, we present them in increasing order of time elapsed. The second and third waves were closest in time, with an average time interval of 2.04 years; the first and second waves were, on average, 3.87 years apart, whereas the first and third waves were 5.90 years apart. Note that sample sizes were not the same for the three correlations. Wave 3 included 132 people; thus, the correlations between the second and third waves (labeled 2 and 3 ) and the first and third waves ( 1 and 3 ) included 132 people. The remaining Wave 1-Wave 2 correlation (1 and 2) included all 208 participants from the second wave.

Table 2

Time-Lagged Correlations Within and Between Perceptual Speed Composite Scores and Knowledge Composite Scores as a Function of Distance Between Measurement Occasions

\begin{tabular}{lcccc}
\hline & \multicolumn{3}{c}{ Measurement occasions } \\
\cline { 2 - 4 } \multicolumn{1}{c}{ Descriptive index } & 2 and 3 & 1 and 2 & 1 and 3 \\
\hline Average time interval (years) & 2.04 & 3.87 & 5.90 \\
$n$ (pairwise deletion) & 132 & 208 & 132 \\
Correlations & & & \\
$\quad$ Earlier speed with later speed & .78 & .69 & .68 \\
$\quad$ Earlier knowledge with later knowledge & .76 & .79 & .74 \\
$\quad$ Earlier speed with later knowledge & .51 & .48 & .46 \\
$\quad$ Earlier knowledge with later speed & .37 & .32 & .29 \\
\hline
\end{tabular}

Note. The concurrent correlation between perceptual speed and knowledge averaged over the three measurement occasions was .53. All correlations differ reliably from zero $(p<.01)$.
As one can immediately observe, the auto-correlations-that is, the test-retest stability coefficients - of both cognitive composite scores are quite high. The differential pattern in longitudinal correlations is displayed in the lagged across-domain indexes (rows 5 and 6 of Table 2). At all three intervals, the correlations of earlier measures of perceptual speed with later measures of knowledge are greater than otherwise. The corresponding pairs of correlations are .51 and .37 for the shortest interval, .48 and .32 for the middle interval, and .46 and .29 for the longest interval.

The message of Table 2 seems clear: Although knowledge correlates with itself in time at least as strongly as perceptual speed does, the cross-lagged correlations that give temporal priority to perceptual speed appear to be greater than the correlations that give temporal priority to knowledge. Therefore, one's current perceptual speed score seems to be a stronger predictor of one's subsequent knowledge score than one's current knowledge score is of one's subsequent perceptual speed score, regardless of the length of the time interval considered. This correlational pattern is, however, liable to technical criticism (Rogosa, 1980a). Nevertheless, we include these descriptive indexes as a first attempt to address the dynamic assumption of the dedifferentiation hypothesis as previously formulated. The BDCSM addresses this hypothesis in a formal and statistically more defendable manner and provides analogous results.

\section{Univariate Change Models}

We dealt with incomplete data patterns by using structural equation modeling software capable of raw data maximum likelihood analyses (Arbuckle, 1996; Enders, 2001). For these analyses, we used the Mx software (Neale, Boker, Xie, \& Maes, 1999). This estimation procedure, which does not impute data but analyzes all existing data, compares each individual's observed data with the relevant portion of the overall expected variance-covariance matrix and mean vector estimated by the model's parameters. In this analysis, an individual misfit is calculated, and the overall sum of misfits (i.e., deviance scores or $-2 \log$-likelihood [LL]) is provided for each model. Table 3 presents the parameter estimates and fit indices of the univariate DCSMs when applied to both the perceptual speed and the knowledge composite scores.

The DCSM postulates that the parameter $\alpha$ be fixed at 1 and that $\beta$ be estimated. For perceptual speed, $\beta$ is .02, which represents a small but positive effect on change scores as previous scores increase. The average of the initial intercept is 59.08, whereas the yearly average decrease in latent difference scores, implied by the negative mean of the slope factor, is -1.64 . This average decrease cannot be interpreted directly as yearly decreases in average scores, because each score is affected not only by the latent slope factor but also by the previous score (through the auto-proportion parameter $\beta$ ). Moreover, the effects of the dynamic parameter at each age are compounded by the effects at all previous times, so straightforward interpretations of the parameter estimates are not simple (see Equations 4 and 6). Individual differences are represented with respect to the initial intercept $\left(S D_{L}=6.43\right)$, the slope score $\left(S D_{S}=0.09\right)$, the residual score $\left(S D_{R}=4.69\right)$, and the correlation intercept-slope $\left(R_{L S}=-.99\right)$. The DCSM for perceptual speed obtains an overall misfit index (-2LL) of 5,820 for seven estimated parameters. Analogous results are presented for the knowledge construct. For knowledge, the DCSM postulates a 
Table 3

Parameter Estimates and Fit Statistics of the Univariate DualChange Score Model Applied Separately to Perceptual Speed and Knowledge

\begin{tabular}{lcc}
\hline & \multicolumn{2}{c}{ Composite score } \\
\cline { 2 - 3 } Parameter estimate & Speed & Knowledge \\
\hline Mean level factor & 59.08 & 54.23 \\
Mean slope factor & -1.64 & -3.29 \\
$S D$ level factor & 6.43 & 8.18 \\
$S D$ slope factor & 0.09 & 0.48 \\
$r$ level-slope factors & -.99 & -.99 \\
$S D$ uniqueness & 4.69 & 3.97 \\
$\beta$ & .02 & .06 \\
Misfit (-2LL) & 5,820 & 5,832 \\
No. of parameters & 7 & 7 \\
\hline
\end{tabular}

Note. $\quad-2 \mathrm{LL}$ (log-likelihood) is the deviance measure of misfit; the mean level factor refers to level of functioning at the age of 70 years.

slightly stronger positive auto-proportion coefficient $\beta$ (.06), whereas the mean of the slope factor is greater than for perceptual speed (-3.29). Thus, for knowledge, the additive component is more negative than it is for perceptual speed, but this is counterbalanced by the more positive multiplicative component $\beta$. In both cases, the correlation between the level and slope factors is empirically underidentified (i.e., it is estimated at its preset lower boundary). This does not affect the remaining parameter estimates of the model, and as such we dismiss it (i.e., fixing the correlation to other values such as 0 or .99 did not significantly alter the fit or the other parameter estimates of the model).

Table 4 presents the summary fit indexes of the alternative univariate change models and the change in fit relative to the fully parameterized (i.e., dual-change score) model. As discussed previously, all other change models tested here are nested within the DCSM, and as such direct statistical comparisons of any change model with the DCSM are legitimate. To assess the difference in fit, we computed the difference in $-2 \mathrm{LL}$ deviance misfit. Such differences can be compared asymptotically with a chi-square distribution with as many degrees of freedom as the difference in the number of parameters estimated by the two models compared (McArdle \& Hamagami, 1996; Rasbash et al., 2000). We then reexpressed the difference in fit statistics as the root-mean-square error of approximation (RMSEA; Steiger \& Lind, 1980, as cited in Browne \& Cudeck, 1993). The RMSEA can be used as a measure of absolute fit as well as comparative fit (Browne \& DuToit, 1992). We applied the RMSEA fit index solely for comparative purposes, and hence we refer to it as the comparative RMSEA (CRMSEA). In comparisons of two nested models, a resulting CRMSEA of 0 means that there is no reliable (i.e., significant) difference between the fits of the models (at which point the most parsimonious model is usually recommended). Values of 0.05 or less can be interpreted as there being almost no statistical difference between the fits of the two models. Hence, the statistical difference between the two fits is significant, but only slightly so. In that case, the two models represent the structure of the data almost equally well.

For perceptual speed, the DCSM appears to be the best-fitting model of the four tested. The decrease in misfit when moving from a proportional change model to a DCSM is evident (drop in chi-square value of 20 for 3 degrees of freedom, CRMSEA = 0.105), whereas the drop in misfit from the no change score model to the DCSM is very large (change in chi-square value of 209 for 4 degrees of freedom, CRMSEA $=0.315$ ). However, the difference in fit between the CCSM and the DCSM is not major $($ CRMSEA $=0.044)$. Thus, the proportion parameter $\beta$ (the additional parameter of the DCSM over the CCSM), although statistically reliable, is not strong. For knowledge, the supremacy of the DCSM tested on these data is evident in all three model comparisons. The differences in comparison chi-square values of the constant, proportional, and no change models with the DCSM are 8,19 , and 72 units, respectively (for 1, 3, and 4 degrees of freedom, in that order).

From the parameter estimates of each change model, longitudinal expectations can be calculated for the predicted factor scores (as in McArdle \& Hamagami, 1999). These represent the yearly mean values of the sample examined from 70 to 104 years of age predicted by the change models. Figure 6 plots the population true score expectations predicted by the four change models for the perceptual speed composite variable. Figure 7 plots the longitudinal expectations for the knowledge composite variable. In both figures, the trajectory of the no change score model is represented with triangles; the proportional change score model, with squares; the CCSM, with asterisks; and the DCSM, with circles.

\section{Bivariate Change Models}

This section reports the application of the BDCSM to test for dynamic structural relations between perceptual speed and knowledge. The saturated multivariate model, as presented in Figure 5, estimates a total of 20 parameters (the 7 parameters of each univariate model, the 4 intervariable correlations among the two level and slope factors of perceptual speed with the two level and slope factors of knowledge, and the 2 cross-lagged regression paths). The parameters of major interest when examining dynamic relationships between the two time series are the proportion parameters within each time series (i.e., $\beta$ ) and across the two time series (i.e., $\gamma$ ). As such, the different hypotheses developed to test

\section{Table 4}

Fit Statistics of Dual-Change, Constant Change, Proportional Change, and No Change Score Models Applied to Perceptual Speed and Knowledge and Associated Fit Comparisons With the Dual-Change Score Model

\begin{tabular}{|c|c|c|c|c|c|c|}
\hline \multirow[b]{2}{*}{ Model $(\Delta d f)$} & \multicolumn{3}{|c|}{ Speed } & \multicolumn{3}{|c|}{ Knowledge } \\
\hline & $-2 \mathrm{LL}$ & $\Delta-2 \mathrm{LL}$ & CRMSEA & $-2 \mathrm{LL}$ & $\Delta-2 \mathrm{LL}$ & CRMSEA \\
\hline Dual change & 5,820 & & & 5,832 & & \\
\hline $\begin{array}{l}\text { Constant change } \\
\text { (1) }\end{array}$ & 5,822 & 2 & 0.044 & 5,840 & 8 & 0.116 \\
\hline $\begin{array}{l}\text { Proportional } \\
\text { change ( } 3 \text { ) }\end{array}$ & 5,840 & 20 & 0.105 & 5,851 & 19 & 0.102 \\
\hline No change (4) & 6,029 & 209 & 0.315 & 5,904 & 72 & 0.182 \\
\hline
\end{tabular}

Note. The no, proportional, and constant change score models are all nested within the dual-change score model, so direct statistical model comparisons are possible. -2LL (log-likelihood) is the deviance measure of misfit. CRMSEA = comparative root-mean-square error of approximation. 


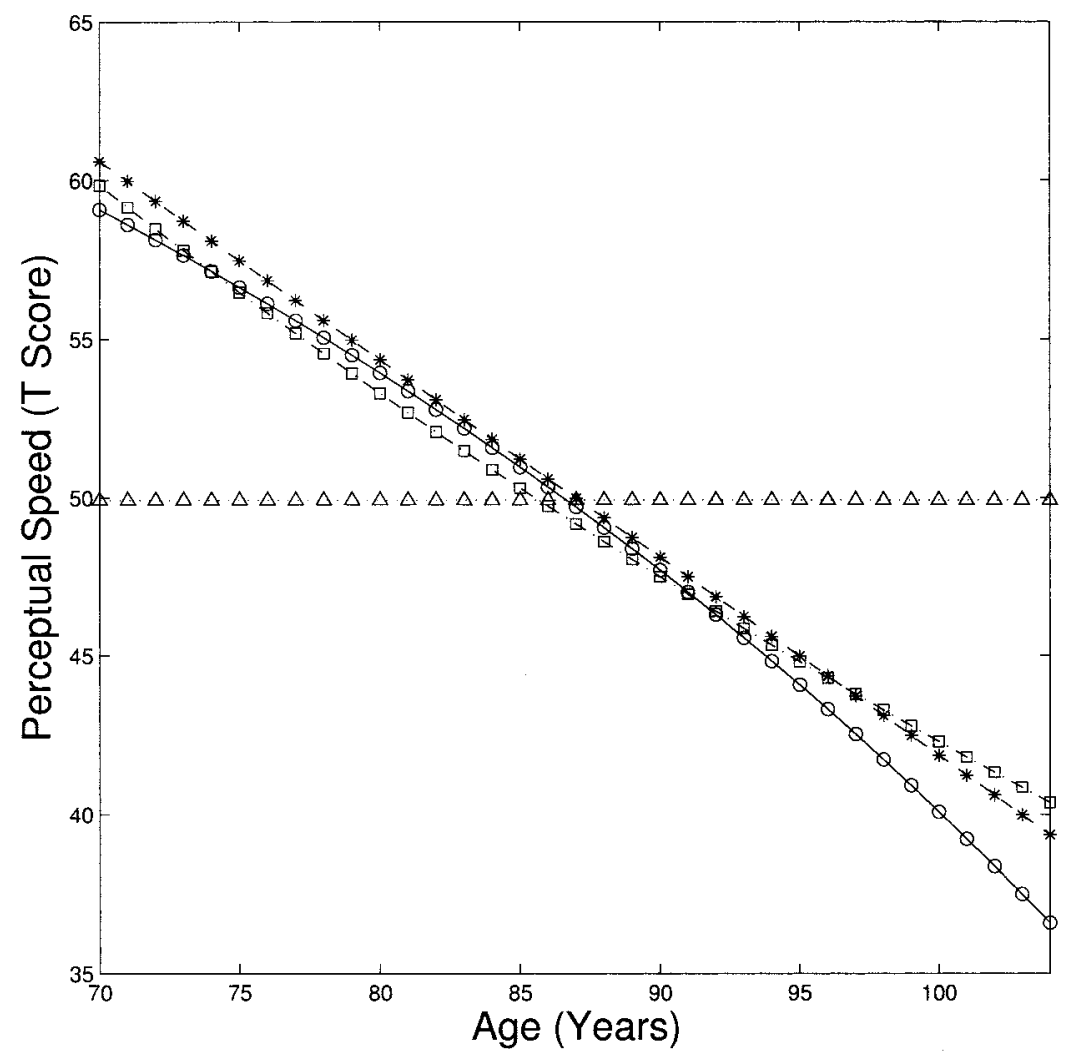

Figure 6. Expected mean population factor scores for perceptual speed as predicted by the dual-change score model (represented by circles), constant change score model (represented by asterisks), proportional change score model (represented by squares), and no change score model (represented by triangles). The best-fitting models are the dual-change and constant change score models (see Table 4).

the dynamic relationships of the two variables can be expressed with structural models that are alternative versions of the saturated BDCSM. That is, all tested versions of the BDCSM differ only with respect to the dynamic parameters $\beta$ and $\gamma$. Because all bivariate models tested are nested within the saturated BDCSM estimating 20 parameters, direct statistical comparisons are possible between any bivariate model tested and the saturated BDCSM.

For simplicity, all BDCSM results reported in Tables 5 and 6 are limited to presentation of dynamic parameters and the total misfit index of each model. In Table 5, the saturated BDCSM is presented first. This model estimates the auto-proportion parameters of both time series (i.e., $\beta_{S}$ and $\beta_{K}$ ) as well as the two across-time series cross-lagged effects (i.e., $\gamma_{S \rightarrow \Delta K}$ and $\gamma_{K \rightarrow \Delta S}$ ). The remaining bivariate models are restrictions of this saturated BDCSM in which one or more of the four dynamic parameters listed here are constrained (the parameters examined are either set to be equal to zero or set to be equal to each other).

Table 5 concerns the analyses aimed at refining the individual time series within the context of the bivariate model. When the two time series were tested independently, it was concluded that two different models might best represent the data of the two cognitive constructs analyzed. The time series for perceptual speed displayed a weak auto-proportion parameter $\beta$. A more parsimonious CCSM obtained a fit close to the fit of a less parsimonious DCSM. Thus, it might be argued that a CCSM suffices to represent the repeated perceptual speed measures. The time series of knowledge, on the other hand, displayed a reliable auto-proportion parameter, such that the less parsimonious DCSM was a better representation of the knowledge data than was a CCSM. We first ascertained whether the dynamic idiosyncracies of the two time series are reflected in the same manner in the context of bivariate relationships. As such, the models in Table 5 tested the different configurations of the within-construct dynamic parameters $\beta$ while always allowing for the estimation of the between-constructs dynamic parameters $\gamma$. The four models were as follows: (a) auto-proportionality present in both time series (i.e., both $\beta_{S}$ and $\beta_{K}$ are estimated; this model is the saturated BDCSM), (b) only knowledge displaying autoproportionality ( $\beta_{S}$ is fixed at 0 , whereas $\beta_{K}$ is estimated), (c) only perceptual speed displaying auto-proportionality $\left(\beta_{S}\right.$ is estimated, whereas $\beta_{K}$ is fixed at 0 ), and (d) no auto-proportionality present (i.e., both $\beta_{S}$ and $\beta_{K}$ are fixed at 0 ). All four models estimate both cross-lagged regression parameters and differ only with respect to the within-construct auto-proportion $\beta$ parameters.

On the basis of the univariate results of Table 4 , one might expect the "zero speed auto-proportion" model (with $\beta_{S}=0$ and $\beta_{K}$ estimated) to be the best-fitting model among the four tested here. We tested the significance of the $\beta_{S}$ parameter and of all dynamic parameters in subsequent analyses by forcing $\beta_{S}$ to zero. Then we compared the new statistical fit index with the previous index when the dynamic parameter of inquiry was estimated (in 


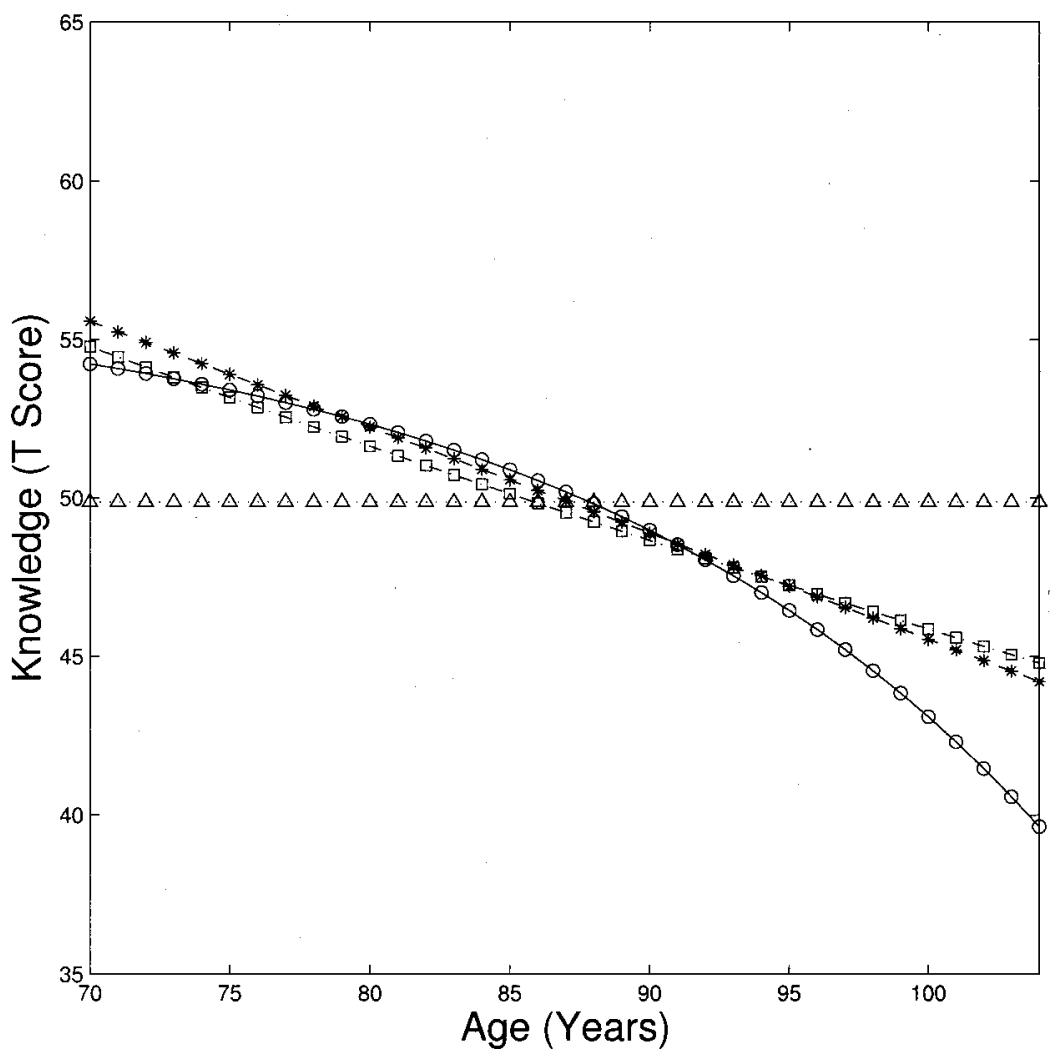

Figure 7. Expected mean population factor scores for knowledge as predicted by the dual-change score model (represented by circles), constant change score model (represented by asterisks), proportional change score model (represented by squares), and no change score model (represented by triangles). The best-fitting model is the dual-change score model (see Table 4).

this analysis, $\beta_{S}$ ). The change in fit of the two nested models represents the statistical significance test of the dynamic parameter examined. For $\beta_{S}$, the comparison resulted in a change in chisquare fit of 3 with 1 degree of freedom (represented by the dynamic parameter). According to the resulting CRMSEA (0.062), the saturated dynamic model and the zero speed auto-proportion model were statistically different from each other, which justifies estimation of the additional $\beta_{S}$ parameter. Analogously, enforcing the auto-proportionality parameter for knowledge to be zero ("zero knowledge auto-proportion") resulted in a considerable loss of fit $($ CRMSEA $=1.211)$. When the dynamic parameters $\beta$ of both time series were forced to zero ("neither auto-proportion"), a significant loss of fit was again observed (CRMSEA $=0.904)$. Thus, we conclude that estimation of both $\beta_{S}$ and $\beta_{K}$ is statistically justified. In the bivariate context, both perceptual speed and knowledge display reliable auto-proportionality. Note that the estimation of $\beta_{K}$ resulting in a value of -0.99 is legitimate, in that this parameter is an unstandardized regression weight and, as such, is not bounded to the convenient range of -0.99 to 0.99 .

Having ascertained the existence of statistically reliable autoproportionality parameters for both knowledge and perceptual speed in the bivariate context, we may now tackle the questions concerning the dynamic relationships between the two constructs. In each analysis to follow, we apply the same single parameter testing paradigm as we did earlier (we set the parameters of interest to zero and ascertain the consequent drop in statistical fit). In Table 6, five versions of the BDCSM are applied. In the first, both cross-lagged dynamic effects are estimated (i.e., "coupling effect"; both $\gamma_{\text {speed } \rightarrow \Delta \text { knowledge }}$ and $\gamma_{\text {knowledge } \rightarrow \Delta \text { speed }}$ are estimated). This model postulates the full coupling effect between the two time series. Both variables are driving the system, and there is no unique leading indicator within the system considered. In the second version, perceptual speed is the sole leading indicator of the system ("speed as leader"; $\gamma_{\text {speed } \rightarrow \Delta \text { knowledge }}$ is fixed at 0 and $\gamma_{\text {knowledge } \rightarrow \Delta \text { speed }}$ is estimated). In the third, knowledge is the sole leading indicator of the system ("knowledge as leader"; $\gamma_{\text {speed } \rightarrow \Delta \text { knowledge }}$ is estimated and $\gamma_{\text {knowledge } \rightarrow \Delta \text { speed }}$ is fixed at 0 ). The fourth version is "equal leading effects" $\left(\gamma_{\text {speed } \rightarrow \Delta \text { knowledge }}\right.$ is estimated to be equal to $\left.\gamma_{\text {knowledge } \rightarrow \Delta \text { speed }}\right)$. Finally, in the fifth version, no cross-lagged dynamic effects are estimated (i.e., "no leading effects"; both $\gamma_{\text {speed } \rightarrow \Delta \text { knowledge }}$ and $\gamma_{\text {knowledge } \rightarrow \Delta \text { speed }}$ are fixed at 0). Analogously to the previous analyses, this series of BDCSMs presents a convenient situation of nested models. The first (saturated, coupling effect) model is the least parsimonious model and estimates both cross-lagged regression effects; the remaining four models are nested within it. This allows for the statistical tests of hypotheses presented in the first column of Table 6

The comparison of the no leading effect model with the coupling effect model is equivalent to the statistical test of the two $\gamma$ 
Table 5

Alternative Hypotheses Regarding the Auto-Proportion Parameter $\beta$ of the Bivariate Dual-

Change Score Model With Relative Fit Statistics and Difference in Fit Relative to the Saturated Dynamic (Bivariate Dual-Change Score) Model

\begin{tabular}{|c|c|c|c|c|c|c|}
\hline Hypothesis & $\begin{array}{l}\text { Parameter constraints } \\
\text { in model }\end{array}$ & $\beta / \gamma$ & Misfit & $\Delta-2 \mathrm{LL}$ & $\Delta d f$ & CRMSEA \\
\hline \multirow{2}{*}{$\begin{array}{l}\text { Saturated dynamic } \\
\text { model }\end{array}$} & $\beta_{S}=? / \gamma_{S \rightarrow \Delta K}=?$ & $-0.04 / 0.51$ & \multirow[t]{2}{*}{12,314} & & & \\
\hline & $\beta_{K}=? / \gamma_{K \rightarrow \Delta S}=?$ & $-0.99 / 0.07$ & & & & \\
\hline \multirow{2}{*}{$\begin{array}{l}\text { Zero speed auto- } \\
\text { proportion }\end{array}$} & $\beta_{S}=0 / \gamma_{S \rightarrow \Delta K}=?$ & $0 / 0.50$ & \multirow[t]{2}{*}{12,317} & \multirow[t]{2}{*}{3} & \multirow[t]{2}{*}{1} & \multirow[t]{2}{*}{0.062} \\
\hline & $\beta_{K}=? / \gamma_{K \rightarrow \Delta S}=?$ & $-1.01 / 0.04$ & & & & \\
\hline \multirow{2}{*}{$\begin{array}{l}\text { Zero knowledge } \\
\text { auto-proportion }\end{array}$} & $\beta_{S}=? / \gamma_{S \rightarrow \Delta K}=?$ & $-1.48 /-3.09$ & \multirow[t]{2}{*}{13,070} & \multirow[t]{2}{*}{756} & \multirow[t]{2}{*}{1} & \multirow[t]{2}{*}{1.211} \\
\hline & $\beta_{K}=0 / \gamma_{K \rightarrow \Delta S}=?$ & $0 / 0.25$ & & & & \\
\hline \multirow{2}{*}{$\begin{array}{l}\text { Neither auto- } \\
\text { proportion }\end{array}$} & $\beta_{S}=0 / \gamma_{S \rightarrow \Delta K}=?$ & $0 /-0.45$ & \multirow[t]{2}{*}{13,158} & \multirow[t]{2}{*}{844} & \multirow[t]{2}{*}{2} & \multirow[t]{2}{*}{0.904} \\
\hline & $\beta_{K}=0 / \gamma_{K \rightarrow \Delta S}=?$ & $0 /-0.05$ & & & & \\
\hline
\end{tabular}

Note. The zero speed, zero knowledge, and neither auto-proportion models are all nested within the saturated dynamic model, so direct, statistical model comparisons are possible. $\beta_{S}$ and $\beta_{K}$ are the auto-proportion parameters $\beta$ of perceptual speed and knowledge, respectively; $\gamma_{S \rightarrow \Delta K}$ and $\gamma_{K \rightarrow \Delta S}$ are the cross-lagged regressive parameters of earlier perceptual speed on later knowledge and earlier knowledge on later perceptual speed, respectively; $=$ ? means that the parameter is estimated; $=0$ means that the parameter is fixed at $0 ; \Delta-2 \mathrm{LL}$ (log-likelihood) is the difference in the deviance measure of misfit; $\Delta d f$ is the difference in degrees of freedom. CRMSEA $=$ comparative root mean squared error of approximation.

parameters. This comparison results in a chi-square value of $12,349-12,314=35$ with 2 degrees of freedom, or a CRMSEA of 0.179 (fifth row of Table 6). As such, we may conclude that there is some sort of reliable dynamic feature present that links the two composite variables analyzed (i.e., the coupling effect model, although less parsimonious, better describes the structure of the data).

By setting $\gamma_{\text {speed } \rightarrow \Delta \text { knowledge }}$ to zero (second row of Table 6), we obtain a misfit index of 12,345. Comparing this model with the coupling effects model corresponds to testing the

Table 6

Alternative Hypotheses Regarding the Cross-Lagged Parameter $\gamma$ of the Bivariate Dual-Change Score Model With Relative Fit Statistics and Difference in Fit Relative to the Saturated Dynamic (Bivariate Dual-Change Score) Model

\begin{tabular}{|c|c|c|c|c|c|c|}
\hline Hypothesis & Model & $\beta / \gamma$ & $\begin{array}{c}\text { Misfit } \\
(-2 L L)\end{array}$ & $\Delta-2 \mathrm{LL}$ & $\Delta d f$ & CRMSEA \\
\hline Coupling effect & $\begin{array}{l}\beta_{S}=? / \gamma_{S \rightarrow \Delta K}=? \\
\beta_{K}=? / \gamma_{K \rightarrow \Delta S}=?\end{array}$ & $\begin{array}{l}-0.04 / 0.51 \\
-0.99 / 0.07\end{array}$ & 12,314 & & & \\
\hline Speed as leader & $\begin{array}{l}\beta_{S}=? / \gamma_{S \rightarrow \Delta K}=0 \\
\beta_{K}=? / \gamma_{K \rightarrow \Delta S}=?\end{array}$ & $\begin{array}{l}-0.01 / 0 \\
-0.98 / 0.03\end{array}$ & 12,345 & 31 & 1 & 0.241 \\
\hline Knowledge as leader & $\begin{array}{l}\beta_{S}=? / \gamma_{S \rightarrow \Delta K}=? \\
\beta_{K}=? / \gamma_{K \rightarrow \Delta S}=0\end{array}$ & $\begin{array}{l}0.02 / 0.48 \\
-1.02 / 0\end{array}$ & 12,324 & 10 & 1 & 0.132 \\
\hline Equal leading effects & $\begin{array}{l}\beta_{S}=? / \gamma_{S \rightarrow \Delta K}=? \\
\beta_{K}=? / \gamma_{K \rightarrow \Delta S}=?\end{array}$ & $\begin{array}{l}-0.02 / 0.08 \\
-0.97 / 0.08\end{array}$ & 12,339 & 25 & 1 & 0.216 \\
\hline No leading effects & $\begin{array}{l}\beta_{S}=? / \gamma_{S \rightarrow \Delta K}=0 \\
\beta_{K}=? / \gamma_{K \rightarrow \Delta S}=0\end{array}$ & $\begin{array}{r}0.01 / 0 \\
-1.00 / 0\end{array}$ & 12,349 & 35 & 2 & 0.179 \\
\hline
\end{tabular}

Note. The speed as leader, knowledge as leader, equal leading effects, and no leading effects models are all nested within the coupling effect model, so direct, statistical model comparisons are possible. $\beta_{S}$ and $\beta_{K}$ are the auto-proportion parameters $\beta$ of perceptual speed and knowledge, respectively; $\gamma_{S \rightarrow \Delta K}$ and $\gamma_{K \rightarrow \Delta S}$ are the cross-lagged regressive parameters of earlier perceptual speed on later knowledge and earlier knowledge on later perceptual speed, respectively; $=$ ? means that the parameter is estimated; $=0$ means that the parameter is fixed at $0 ; \Delta-2 \mathrm{LL}$ (log-likelihood) is the difference in the deviance measure of misfit; $\Delta d f$ is the difference in degrees of freedom. CRMSEA = comparative root mean squared error of approximation. 
$\gamma_{\text {speed } \rightarrow \Delta \text { knowledge }}$ parameter. That is, the hypothesis that perceptual speed is the leading indicator of the bivariate system is statistically tested in this fashion. The comparison yields a loss in fit (chi-square) from the saturated coupling effect model of $12,345-12,314=31$ with 1 degree of freedom. The corresponding CRMSEA is 0.241, which speaks to a definite loss in fit. Analogously, by postulating that there is no effect from knowledge to the subsequent change in perceptual speed (i.e., $\gamma_{\text {knowledge } \rightarrow \Delta \text { speed }}=0$; third row in Table 6), the misfit index obtained is 12,324 . This parameter constraint caused a loss in chi-square fit of $10(12,324-12,314)$ with 1 degree of freedom. The resulting CRMSEA is 0.132 , smaller than the CRMSEA testing the "speed as leader" hypothesis. Finally, and most important, the equal leading effect model forces the cross-lagged regression parameters $\gamma$ to be equal, so this model postulates each variable to affect the change of the other variable with equal magnitude. The misfit of this model is 12,339 , and the comparison with the full model results in a loss in fit equivalent to a chi-square value of $25(12,339-12,314)$ with 1 degree of freedom, corresponding to a CRMSEA of 0.216 . Thus, we can conclude that the two cross-lagged regression weights differ reliably in magnitude. Specifically, within the system of structural relations considered in the present study, perceptual speed exerts a stronger dynamic effect on knowledge than knowledge does on perceptual speed.

\section{Discussion}

The present article had two goals, one substantive and the other methodological. The substantive goal was to examine empirically and test formally the structural dynamics of ability dedifferentiation in late-life cognition. The methodological goal was to familiarize readers with a recently developed variant of latent growth curve modeling that enables researchers to test dynamic structural hypotheses on the basis of incomplete and imbalanced longitudinal data (Bell, 1953, 1954). In the following sections, each of the two goals is addressed in turn.

\section{Main Finding of the Present Study}

In line with our guiding hypothesis, the analyses reported in the present study indicate that the age-associated influence of perceptual speed on changes in knowledge is greater than the ageassociated influence of knowledge on changes in perceptual speed. This is seen most clearly when the freely estimated parameters of the BDCSM are inserted into the prediction equations for the BDCSM (Equations 4 and 6). Specifically, we obtain

$$
\begin{aligned}
\widehat{\text { Speed }}_{t}=(0.96) \cdot \widehat{\text { Speed }}_{t-1} & \\
& +(0.07) \cdot \widehat{\text { Knowledge }}_{t-1}+(-2.05)
\end{aligned}
$$

and

$$
\begin{aligned}
\widehat{\text { Knowled }}_{t}=(0.01) \cdot \widehat{\text { Knowledge }}_{t-1} \\
+(0.51) \cdot \widehat{\text { Speed }}_{t-1}+(24.01)
\end{aligned}
$$

where the hat symbol signifies the true, latent score of the cognitive ability (i.e., without unrelated residual or error variance). As is evident on the basis of these equations, perceptual speed dominates predictions in terms of both abilities.

\section{Substantive Implications}

Our substantive goal was to test the hypothesis that ability dedifferentiation, defined as the convergence of age gradients and increments in covariance between mechanic and pragmatic abilities, is brought about by negative age changes in the mechanics of cognition. Perceptual speed was used to index mechanics, and knowledge was used to index pragmatics. The results were fully consistent with the hypothesis. Under the assumption that perceptual speed and knowledge can be taken to represent mechanic (broad fluid) and pragmatic (broad crystallized; cf. Horn, 1989) domains of cognition, the present findings reveal, perhaps for the first time, the structural dynamics of dedifferentiation in late-life cognition.

To use a hydraulic metaphor, Equations 9 and 10 can be interpreted as a dynamic system of "variance pumps." The cross-lagged gamma parameters specify how much variance of a given domain is pumped into changes in the other domain. Put metaphorically, then, our analyses show that a comparatively large amount of perceptual speed variance is pumped into changes in knowledge. In contrast, a comparatively small amount of knowledge variance is pumped into changes in perceptual speed. In the BDCSM, variance in a given ability is modeled as the additive combination of its own variance at the preceding time point and its change variance. It follows that knowledge, through its own changes, acts as a recipient of variance in perceptual speed. Thus, the influence of perceptual speed on changes in knowledge slowly transforms the very nature of knowledge in old and very old age. With advancing age, variance in knowledge is increasingly dominated by variance in perceptual speed. If generalized to mechanic and pragmatic abilities at large, our results suggest that nominally pragmatic abilities are increasingly governed by mechanic variance. Thus, in line with earlier interpretations of cross-sectional data (e.g., Baltes \& Lindenberger, 1997; Lindenberger \& Baltes, 1994, 1997), the dedifferentiation of age gradients and covariance structures between pragmatic and mechanic abilities can be construed as the consequence of an age-associated "mechanization" of pragmatic abilities.

In the meantime, the present pattern of results has been replicated by Ghisletta and de Ribaupierre (2003) in the Swiss Interdisciplinary Longitudinal Study on the Oldest Old (SWILSO-O; Lalive d'Epinay, Pin, \& Spini, 2001), a parallel-cohort, longitudinal study involving two samples between 80 and 85 years of age at study inception. In the second cohort of the SWILSO-O, assessed three times so far, asymmetric dynamic relations over an average period of 1.05 years were observed between the Cross Out test (Woodcock \& Bonner Johnson, 1990; assumed to be a marker of broad-Gf, or mechanic cognitive abilities) and the verbal fluency test (Cardebat, Doyon, Puel, Goulet, \& Joanette, 1990; assumed to be a marker of broad-Gc, or pragmatic cognitive abilities). Changes in the pragmatic ability marker were predicted by previous states of mechanic ability, whereas the opposite was not true. Note that the ratio of interindividual differences in initial age over intraindividual changes in age was much smaller in the SWILSO-O than in the BASE. Thus, the presence of asymmetric dynamic relations in both studies, despite notable differences in 
sampling schemes, suggests that the pattern of findings observed with the BASE sample was not induced by an undue influence of cross-sectional information.

A further prediction based on the foregoing considerations and some additional assumptions is that nominally pragmatic abilities, rather than mechanic abilities, may act as ultimate (end-of-life) indicators of mechanic decline. In the present study, the structural dynamics between perceptual speed and knowledge were modeled as a function of distance from birth (i.e., chronological age). However, in old and very old age, distance from birth and distance to death are highly correlated (e.g., mortality increases with age). Thus, the proposed mechanization of pragmatic abilities may, in part, form a component of an end-of-life syndrome (Anstey, Luszcz, Giles, \& Andrews, 2001; Baltes, Schaie, \& Nardi, 1971; Maier \& Smith, 1999; cf. Riegel \& Riegel, 1972). Whereas mechanic decline in nominally mechanic abilities is normative across the entire adult life span, mechanic decline in nominally pragmatic abilities may be the signature of impending death. Initial BASE longitudinal results appear to be consistent with this hypothesis (Singer et al., 2003).

\section{Methodological Considerations}

Strengths of the present dynamic approach. This article has advocated a structural and dynamic approach to the multivariate analysis of incomplete longitudinal data. In our view, this approach offers a number of distinct methodological advantages but also involves some problems and limitations. The advantages fall into two categories: those related to more general, well-known properties of structural equation modeling and those that are more specific to the dynamic (time-based) properties of the variant used in this study. The following properties seem particularly relevant: (a) formal specification and testing of structural and time-based (dynamic) hypotheses within and between two sets of variables; (b) representation of change at the level of true scores, which enhances the interpretability of cross-lagged effects (e.g., Rogosa, 1980b); (c) reformulation of classical models of change (e.g., Markov simplex model with proportional parameters and linear growth models with individual differences) within the structural equation framework; and (d) accommodation of variable patterns and degrees of incomplete data. Taken together, these benefits underscore the utility of the BDCSM for the dynamic study of structural change with incomplete longitudinal data.

Violations of assumptions. At the same time, the present application of DCSMs is not without limitations and problems. The most important problem concerns violations of statistical assumptions. To yield unbiased and interpretable parameter estimates, applications of structural equation modeling generally require sample homogeneity and random data incompleteness (McArdle, 1994; Rubin, 1974). With respect to sample homogeneity, the unimodal and symmetrical shape of the distribution of individuals' contributions to the overall $-2 \mathrm{LL}$ misfit statistic for the fully parameterized BDCSM suggests that model parameters were not strongly influenced by a few individuals with very large residuals. However, from a conceptual point of view, it seems likely that the present sample was not homogeneous in nature but comprised different forms of aging. Future analyses need to include empirical markers of heterogeneity (e.g., dementia status and cardiovascular disease) to examine their moderating effect on the structural dy- namics observed in the present study. Variants of the DCSM incorporating covariates are feasible (Ghisletta \& Lindenberger, 2003; McArdle et al., 2003).

Furthermore, the assumption of random data incompleteness is clearly inconsistent with the data analyzed in the present study. As reported earlier, higher levels of functioning in both perceptual speed and knowledge were related to lower mortality and to higher participation rates among survivors. This finding is not idiosyncratic to the present study. Instead, the nonrandom nature of old-age mortality and longitudinal study participation is ubiquitous (Lindenberger et al., 2002; Siegler \& Botwinick, 1979; for a summary, see Lindenberger et al., 1999). The crucial question in the present context is not so much whether data incompleteness was selective or not (because it clearly was) but whether the existence of nonrandom missing data biased the analyses in favor of finding the observed longitudinal supremacy of perceptual speed over knowledge. Descriptively, the observed Time 1-Time 3 selection effects were slightly larger for perceptual speed than for knowledge (0.774 vs. 0.494 standard deviation units). Thus, selection was not associated with a greater reduction in variance of knowledge than in variance of perceptual speed. On the basis of this observation, it is difficult to see how selection could have conditioned the data in favor of a stronger influence of perceptual speed on knowledge than of knowledge on perceptual speed.

Limitations. An important limitation of the present analyses concerns the sparse sampling of intraindividual longitudinal change. As reported earlier, we used limited longitudinal information (longitudinal observation intervals averaged 1.98 years, with a median of 0.18 years $^{1}$ ) to estimate the structural dynamics of an age period spanning 34 years. Hence, the analyses relied heavily on cross-sectional information in the estimation of a longitudinal process (Bell, 1953, 1954; Duncan, Duncan, \& Hops, 1996; Ghisletta \& McArdle, 2001; McArdle \& Anderson, 1990). In theory, and as shown in Equations 6 and 7, mean cross-sectional changes could provide a nonrejectable, underidentified solution for dynamic parameters at the population level. Empirically, however, further sensitivity analyses of the data set with only Time 1 cross-sectional information did not yield any solutions (e.g., the estimation algorithm implemented with the software used failed to initiate the iteration process with all sets of starting values used because, in the absence of longitudinal data, the DCSM is underidentified). Thus, the dynamic information reported here could not be estimated on the basis of cross-sectional information only.

Conversely, we obtained virtually identical values for the dynamic parameters when we reduced the data set to the subsample of 132 participants assessed at all three occasions and when we analyzed the full sample. Hence, selection did not influence the dynamic parameters (although average levels of perceptual speed and knowledge, for instance, were influenced by selection). Moreover, the replication obtained in a much more age-homogeneous

\footnotetext{
${ }^{1}$ Mean times of assessment (defined with origin zero at the beginning of BASE) were as follows: Time $1,0.13$ years (median time $=0.10$ years, $n=516$ ); Time 2, 3.99 years (median time $=3.98$ years, $n=208$ ); and Time 3, 6.03 years (median time $=6.03$ years, $n=132)$. Overall mean time was 1.98 years (median time $=0.18$ years, $N=856$ ). The overall incongruence between mean and median is reflective of a positively skewed distribution.
} 
sample of very old individuals is very consistent with the present findings (Ghisletta \& de Ribaupierre, 2003). In summary, our control analyses show that the results reported in the present article could be obtained only in the presence of longitudinal information. At the same time, we acknowledge that the dynamic parameters reported here recover both longitudinal and cross-sectional aspects of the data. This property is in line with the general logic of cross-sectional-longitudinal convergence models (Bell, 1953, 1954), which integrate both types of information into overall estimates of population change. Integration of cross-sectional and longitudinal information may produce efficient and unbiased estimates of population change and may qualify as a strength rather than as a limitation of the method, at least if certain assumptions are met. To arrive at a full quantitative understanding of longitudinal and cross-sectional influences on dynamic parameters and the relative importance of assumption violations, future statistical simulations need to vary systematically the relative amount and relative direction of longitudinal and cross-sectional information, the heterogeneity of intraindividual change trajectories, and the degree and kind of nonrandom attrition (see also Hamagami \& McArdle, 2001; McArdle \& Hamagami, 2001).

Finally, we need to acknowledge that cohort and retest effects were left unanalyzed in the present study. Given the absence of an association between sociobiographical indicators and chronological age in the cross-sectional parent sample (see Lindenberger \& Baltes, 1997), our reluctance to examine cohort effects appears permissible. Furthermore, the longitudinal rather than cohortsequential design of the BASE makes it difficult to isolate the influence of retesting with sufficient power and precision (cf. Baltes, Reese, \& Nesselroade, 1988).

\section{Conclusion and Outlook}

The present analyses provide direct empirical support for the widely held assumption that dedifferentiation of interindividual differences in intellectual abilities in old and very old age is a consequence of mechanic constraints. In doing so, these analyses underscore the utility of dynamic modeling techniques in developmental research. Numerous methodological and substantive extensions of the approach pursued in the present article appear possible, but two seem especially timely. The first is methodological in kind and concerns attempts to extend the BDCSM to the trivariate and more-variable case (for a four-variable example, see McArdle et al., 2000, and Ghisletta \& Lindenberger, 2003). Moreover, the possibility of modeling the dynamic parameters as random effects, and hence allowing for individual differences in these parameters, must be considered in future developments. This will allow additional inferences about individual rather than group development. The second extension is substantive and concerns analysis of dynamic relations between perceptual speed and sensory functioning in old age. Given the strong cross-sectional connection between intellectual and sensory functioning in old age (e.g., Lindenberger \& Baltes, 1994), an examination of dynamic relations between the two domains may shed some light on the dimensionality and structure of mechanic decline in old and very old age.

\section{References}

Anstey, K. J., Luszcz, M. A., Giles, L. C., \& Andrews, G. R. (2001). Demographic, health, cognitive, and sensory variables as predictors of mortality in very old adults. Psychology and Aging, 16, 3-11.

Arbuckle, J. L. (1996). Full information estimation in the presence of incomplete data. In G. A. Marcoulides \& R. E. Schumacker (Eds.), Advanced structural equation modeling: Issues and techniques (pp. 243-277). Mahwah, NJ: Erlbaum.

Arminger, G. (1986). Linear stochastic differential equation models for panel data with unobserved variables. In N. Tuma (Ed.), Sociological methodology 1986 (pp. 187-212). San Francisco: Jossey-Bass.

Baltes, P. B. (1987). Theoretical propositions of life-span developmental psychology: On the dynamics between growth and decline. Developmental Psychology, 23, 611-626.

Baltes, P. B. (1997). On the incomplete architecture of human ontogeny: Selection, optimization, and compensation as foundation of developmental theory. American Psychologist, 52, 366-380.

Baltes, P. B., Cornelius, S. W., Spiro, A., Nesselroade, J. R., \& Willis, S. L. (1980). Integration versus differentiation of fluid/crystallized intelligence in old age. Developmental Psychology, 16, 625-635.

Baltes, P. B., \& Lindenberger, U. (1997). Emergence of a powerful connection between sensory and cognitive functions across the adult life span: A new window to the study of cognitive aging? Psychology and Aging, 12, 12-21.

Baltes, P. B., Lindenberger, U., \& Staudinger, U. M. (1998). Life-span theory in developmental psychology. In R. M. Lerner (Ed.), Theoretical models of human development (5th ed., pp. 1029-1143). New York: Wiley.

Baltes, P. B., \& Mayer, K. U. (1999). The Berlin Aging Study: Aging from 70 to 100. Cambridge, England: Cambridge University Press.

Baltes, P. B., Mayer, K. U., Helmchen, H., \& Steinhagen-Thiessen, E. (1993). The Berlin Aging Study (BASE): Overview and design. Ageing and Society, 13, 483-515.

Baltes, P. B., Reese, H. W., \& Nesselroade, J. R. (1988). Life-span developmental psychology: An introduction to research methods. Hillsdale, NJ: Erlbaum.

Baltes, P. B., Schaie, K. W., \& Nardi, A. H. (1971). Age and experimental mortality in a seven-year longitudinal study of cognitive behavior. Developmental Psychology, 5, 18-26.

Bell, R. (1953). Convergence: An accelerated longitudinal approach. Child Development, 24, 145-152.

Bell, R. (1954). An experimental test of the accelerated longitudinal approach. Child Development, 24, 381-386.

Boker, S. M., \& Graham, J. (1998). A dynamical systems analysis of adolescent substance abuse. Multivariate Behavioral Research, 33, 479507.

Boker, S. M., McArdle, J. J., \& Neale, M. (2002). An algorithm for the hierarchical organization of path diagrams and calculation of components of expected covariance. Structural Equation Modeling, 9, $174-$ 194.

Browne, M., \& Cudeck, R. (1993). Alternative ways of assessing model fit. In K. A. Boolen \& J. S. Long (Eds.), Testing structural equation models (pp. 136-162). Newbury Park, CA: Sage.

Browne, M., \& DuToit, S. H. C. (1992). Automated fitting of nonstandard models. Multivariate Behavioral Research, 27, 269-300.

Cabeza, R. (2001). Functional neuroimaging of cognitive aging. In R. Cabeza \& A. Kingstone (Eds.), Handbook of functional neuroimaging of cognition (pp. 331-377). Cambridge, MA: MIT Press.

Cardebat, D., Doyon, B., Puel, M., Goulet, P., \& Joanette, Y. (1990). Evocation formelle et sémantique chez des sujets normaux [Formal and semantic evocation in normal subjects]. Acta Neurologica Belgica, 90, 207-217.

Cattell, R. B. (1971). Abilities: Their structure, growth, and action. Boston: Houghton Mifflin. 
Deary, I. J., Egan, V., Gibson, H. J., Austin, E. J., Brand, C. R., \& Kellaghan, T. (1996). Intelligence and the differentiation hypothesis. Intelligence, 23, 105-132.

Deary, I. J., \& Pagliari, C. (1991). The strength of $g$ at different levels of ability: Have Detterman and Daniel rediscovered Spearman's law of diminishing returns? Intelligence, 15, 251-255.

Duncan, S. C., Duncan, T. E., \& Hops, H. (1996). Analysis of longitudinal data within accelerated longitudinal designs. Psychological Methods, 1 , $236-248$

Ekstrom, R. B., French, J. W., Harman, J. J., \& Dermen, D. (1976). Manual for kit of factor-referenced cognitive tests. Princeton, NJ: Educational Testing Service.

Enders, C. K. (2001). A primer on maximum likelihood algorithms available for use with missing data. Structural Equation Modeling, 8, 128141

Ghisletta, P., \& de Ribaupierre, A. (2003). Support for two-component theories of lifespan cognition from the Swiss Interdisciplinary Longitudinal Study on the Oldest-Old. Manuscript in preparation.

Ghisletta, P., \& Lindenberger, U. (2003). Exploring the structural dynamics of the link between sensory and cognitive functioning in old age: Longitudinal evidence from the Berlin Aging Study. Manuscript in preparation.

Ghisletta, P., \& Lindenberger, U. (in press). Static and dynamic longitudinal structural analyses of cognitive changes in old age. Gerontology.

Ghisletta, P., \& McArdle, J. J. (2001). Latent growth curve analyses of the development of height. Structural Equation Modeling, 8, 531-555.

Guttman, L. (1954). A new approach to factor analysis: The radex. In P. F. Lazarsfeld (Ed.), Mathematical thinking in the social sciences (pp. 258-348). Glencoe, IL: Free Press.

Hamagami, F., \& McArdle, J. J. (2001). Advanced studies of individual differences linear dynamic models for longitudinal data analysis. In G. A. Marcoulides \& R. E. Schumacker (Eds.), New developments and techniques in structural equation modeling (pp. 203-246). Mahwah, NJ: Erlbaum.

Hamagami, F., McArdle, J. J., \& Cohen, P. (2000). A new approach to modeling bivariate dynamic relationships applied to evaluation of comorbidity among DSM-III personality disorder symptoms. In V. J. Molfese \& D. L. Molfese (Eds.), Temperament and personality development across the life span (pp. 253-280). Mahwah, NJ: Erlbaum.

Hertzog, C. (1989). Influences of cognitive slowing on age differences in intelligence. Developmental Psychology, 25, 636-651.

Hertzog, C. (in press). Does longitudinal evidence confirm theories of cognitive aging derived from cross-sectional data? The sample case of processing resource accounts of episodic memory. In R. A. Dixon, L. Bäckman, \& L. G. Nilsson (Eds.), New frontiers in cognitive aging. Oxford, England: Oxford University Press.

Horn, J. L. (1982). The theory of fluid and crystallized intelligence in relation to concepts of cognitive psychology and aging in adulthood. In F. I. M. Craik \& S. Trehub (Eds.), Aging and cognitive processes (pp. 77-117). New York: Plenum.

Horn, J. L. (1989). Models of intelligence. In R. L. Linn (Ed.), Intelligence: Measurement, theory, and public policy: Proceedings of a symposium in honor of Lloyd G. Humphreys (pp. 29-73). Champaign: University of Illinois Press.

Hultsch, D. F., Hertzog, C. A., Dixon, R. A., \& Small, B. J. (1998). Memory change in the aged. Cambridge, England: Cambridge University Press.

Jones, H. E., \& Conrad, H. (1933). The growth and decline of intelligence: A study of a homogeneous group between the ages of ten and sixty. Genetic Psychology Monographs, 13, 223-298.

Lalive d'Epinay, C., Pin, S., \& Spini, D. (2001). Présentation de SWILSO-O, une étude longitudinale suisse sur le grand âge: L'exemple de la dynamique de la santé fonctionnelle [Presentation of SWILSO-O, a longitudinal Swiss study on very old age: The example of the dynamics of functional health]. L'Année Gérontologique, 15, 78-96.

Li, S.-C., \& Lindenberger, U. (1999). Cross-level unification: A computational exploration of the link between deterioration of neurotransmitter systems and dedifferentiation of cognitive abilities in old age. In L.-G. Nilsson \& H. Markowitsch (Eds.), Cognitive neuroscience of memory (pp. 103-146). Toronto, Ontario, Canada: Hogrefe \& Huber.

Lindenberger, U. (2001). Lifespan theories of cognitive development. In N. J. Smelser \& P. B. Baltes (Eds.), International encyclopedia of the social and behavioral sciences (pp. 8848-8854). Oxford, England: Elsevier.

Lindenberger, U., \& Baltes, P. B. (1994). Sensory functioning and intelligence in old age: A strong connection. Psychology and Aging, 9, $339-355$.

Lindenberger, U., \& Baltes, P. B. (1997). Intellectual functioning in old and very old age: Cross-sectional results from the Berlin Aging Study. Psychology and Aging, 12, 410-432.

Lindenberger, U., \& Baltes, P. B. (1999). Die Entwicklungspsychologie der Lebensspanne (Lifespan-Psychologie): Johann Nicolaus Tetens (1736-1807) zu Ehren [Lifespan psychology: In honor of Johann Nicolaus Tetens (1736-1807)]. Zeitschrift für Psychologie, 207, 299-323.

Lindenberger, U., \& Ghisletta, P. (in press). Modeling longitudinal changes in old age: From covariance structures to dynamic systems. In R. A. Dixon, L. Bäckman, \& L.-G. Nilsson (Eds.), New frontiers in cognitive aging. Oxford, England: Oxford University Press.

Lindenberger, U., Gilberg, R. D., Little, T. D., Nuthman, R., Pötter, U., \& Baltes, P. B. (1999). Sample selectivity and generalizability of the results of the Berlin Aging Study. In P. B. Baltes \& K. U. Mayer (Eds.), The Berlin Aging Study: Aging from 70 to 100. Cambridge, England: Cambridge University Press.

Lindenberger, U., Mayr, U., \& Kliegl, R. (1993). Speed and intelligence in old age. Psychology and Aging, 8, 207-220.

Lindenberger, U., Singer, T., \& Baltes, P. B. (2002). Longitudinal selectivity in aging populations: Separating mortality-associated versus experimental components in the Berlin Aging Study. Journals of Gerontology, Series B: Psychological Sciences and Social Sciences, 57B, P474-P482.

MacCallum, R. C., Kim, C., Malarkey, W. B., \& Kiecolt-Glaser, J. K. (1997). Studying multivariate change using multilevel models and latent curve models. Multivariate Behavioral Research, 32, 215-253.

Maier, H., \& Smith, J. (1999). Psychological predictors of mortality in old age. Journals of Gerontology, Series B: Psychological Sciences and Social Sciences, 54B, P44-P54.

McArdle, J. J. (1994). Structural factor analysis experiments with incomplete data. Multivariate Behavioral Research, 29, 409-454.

McArdle, J. J. (2001). A latent difference score approach to longitudinal dynamic structural analyses. In R. Cudeck, S. DuToit, \& D. Sorbom (Eds.), Structural equation modeling: Present and future (pp. 342-380). Lincolnwood, IL: Scientific Software International.

McArdle, J. J., \& Aber, M. S. (1990). Patterns of change within latent variable structural equation models. In A. von Eye (Ed.), Statistical methods in developmental research: Vol. 1. Principles and structuring of change (pp. 151-224). New York: Academic Press.

McArdle, J. J., \& Anderson, E. (1990). Latent variable growth models for research on aging. In J. E. Birren \& K. W. Schaie (Eds.), The handbook of the psychology of aging (3rd ed., pp. 21-44). New York: Plenum.

McArdle, J. J., \& Bell, R. Q. (2000). An introduction to latent growth models for developmental data analysis. In T. D. Little, K. U. Schnabel, \& J. Baumert (Eds.), Modeling longitudinal and multilevel data: Practical issues, applied approaches, and specific examples (pp. 69-107). Mahwah, NJ: Erlbaum.

McArdle, J. J., \& Boker, S. M. (1990). RAMpath: A computer program for automatic path diagrams. Hillsdale, NJ: Erlbaum.

McArdle, J. J., \& Hamagami, F. (1996). Multilevel models from a multiple 
group structural equation perspective. In G. A. Marcoulides \& R. E. Schumaker (Eds.), Advanced structural equation modeling: Issues and techniques (pp. 89-124). Mahwah, NJ: Erlbaum.

McArdle, J. J., \& Hamagami, F. (1999). Latent difference score structural models for linear dynamic analyses with incomplete longitudinal data. In A. Sayer \& L. Collins (Eds.), Methods for the analysis of change (pp. 276-304). Washington, DC: American Psychological Association.

McArdle, J. J., \& Hamagami, F. (2001). Latent difference score structural models for linear dynamic analyses with incomplete longitudinal data. In L. Collins \& A. Sayer (Eds.), New methods for the analysis of change (pp. 137-175). Washington, DC: American Psychological Association.

McArdle, J. J., Hamagami, F., Jones, K., Jolesz, F., Sandor, T., Kikinis, R., et al. (2003). Structural modeling of dynamic changes in memory and brain structure using longitudinal data from the Normative Aging Study. Manuscript submitted for publication.

McArdle, J. J., Hamagami, F., Meredith, W., \& Bradway, K. P. (2000). Modeling the dynamic hypotheses of Gf-Gc theory using longitudinal life-span data. Learning and Individual Differences, 12, 53-79.

McArdle, J. J., \& McDonald, R. P. (1984). Some algebraic properties of the reticular action model for moment structures. British Journal of Mathematical and Statistical Psychology, 27, 234-251.

McArdle, J. J., \& Nesselroade, J. R. (1994). Using multivariate data to structure developmental change. In S. H. Cohen \& H. W. Reese (Eds.), Life-span developmental psychology: Methodological contributions (pp. 223-267). Hillsdale, NJ: Erlbaum.

McArdle, J. J., \& Nesselroade, J. R. (2002). Growth curve analysis in contemporary psychological research. In J. Schinka \& W. Velicer (Eds.), Comprehensive handbook of psychology: Vol. 2. Research methods in psychology (pp. 447-480). New York: Wiley.

McClelland, G. H., \& Judd, C. M. (1993). Statistical difficulties of detecting interactions and moderator effects. Psychological Bulletin, 114, 376-390.

Neale, M. C., Boker, S. C., Xie, G., \& Maes, H. H. (1999). Mx: Statistical modeling (5th ed.). Richmond: Medical College of Virginia.

Nesselroade, J. R., \& Ghisletta, P. (2003). Structuring and measuring change over the lifespan. In U. M. Staudinger \& U. Lindenberger (Eds.), Understanding human development: Dialogues with lifespan psychology (pp. 317-337). Dordrecht, the Netherlands: Kluwer Academic.

Rasbash, J., Browne, W., Goldstein, H., Yang, M., Plewis, I., Healy, M., et al. (2000). A user's guide to MLwiN. London: Institute of Education.

Raudenbush, S. W., \& Chan, W. (1992). Growth curve analysis in accelerated longitudinal designs. Journal of Consulting and Clinical Psychology, 61, 941-951.

Raz, N. (2000). Aging of the brain and its impact on cognitive performance: Integration of structural and functional findings. In F. I. M. Craik $\&$ T. A. Salthouse (Eds.), The handbook of aging and cognition (2nd ed., pp. 1-90). Mahwah, NJ: Erlbaum.

Reinert, G., Baltes, P. B., \& Schmidt, L. R. (1966). Kritik einer Kritik der Differenzierungshypothese der Intelligenz [Critique of a critique of the dedifferentiation hypothesis of intelligence]. Zeitschrift für Experimentelle und Angewandte Psychologie, 13, 602-610.

Riegel, K. F., \& Riegel, R. M. (1972). Development, drop, and death. Developmental Psychology, 6, 309-316.

Rogosa, D. (1980a). Comparisons of some procedures for analyzing longitudinal panel data. Journal of Economics and Business, 32, 136-151.

Rogosa, D. (1980b). A critique of cross-lagged correlations. Psychological Bulletin, 88, 245-258.
Rubin, D. (1974). Characterizing the estimation of parameters in incomplete-data problems. Journal of the American Statistical Association, 69, 467-474.

Rubin, D. C. (1999). Frontal-striatal circuits in cognitive aging: Evidence for caudate involvement. Aging, Neuropsychology, and Cognition, 6, 241-259.

Salthouse, T. A. (1991). Mediation of adult age differences in cognition by reductions in working memory and speed of processing. Psychological Science, 2, 179-183.

Salthouse, T. A., Hambrick, D. Z., Lukas, K. E., \& Dell, T. C. (1996). Determinants of adult age differences on synthetic work performance. Journal of Experimental Psychology: Applied, 2, 305-329.

Schaie, K. W. (1962). A field-theory approach to age changes in cognitive behavior. Vita Humana, 5, 129-141.

Schaie, K. W., Maitland, S. B., Willis, S. L., \& Intieri, R. C. (1998). Longitudinal invariance of adult psychometric ability factor structures across 7 years. Psychology and Aging, 13, 8-20.

Siegler, I., \& Botwinick, J. (1979). A long-term longitudinal study of the intellectual ability of older adults: The matter of selective subject attrition. Journal of Gerontology, 34, 242-245.

Singer, T., Verhaeghen, P., Ghisletta, P., Lindenberger, U., \& Baltes, P. B. (2003). The fate of cognition in very old age: Six-year longitudinal findings in the Berlin Aging Study (BASE). Psychology and Aging, 18, $318-331$.

Steiger, J. H., \& Lind, J. M. (1980, June). Statistically-based tests for the number of common factors. Paper presented at the annual meeting of the Psychometric Society, Iowa City, IA.

Stoolmiller, M. (1994). Antisocial behavior, delinquent peer association, and unsupervised wandering for boys: Growth and change from childhood to early adolescence. Multivariate Behavioral Research, 29, 263 288 .

Tetens, J. N. (1777). Philosophische Versuche über die menschliche Natur und ihre Entwicklung [Philosophical essays on human nature and its development]. Leipzig, Germany: Weidmanns Erben und Reich.

Verhaeghen, P., \& Salthouse, T. A. (1997). Meta-analyses of agecognition relations in adulthood: Estimates of linear and nonlinear age effects and structural models. Psychological Bulletin, 122, 231-249.

Wechsler, D. (1955). Wechsler Adult Intelligence Scale manual. New York: Psychological Corporation.

Wechsler, D. (1982). Handaweisung zum Hamburg-Wechsler-Intelligenz für Erwachsene (HAWIE) [Manual for the Hamburg Wechsler Intelligence Tests for adults]. Bern, Switzerland: Huber.

Wold, H. (1949). A large sample test for moving averages. Journal of the Royal Statistical Society, Series B, 2, 297-305.

Woodcock, R., \& Bonner Johnson, M. (1990). Tests of cognitive ability, standard and supplemental batteries: Examiner's manual. Allen, TX: DLM Teaching Resources.

Wright, S. (1918). On the nature of size factors. Genetics, 3, 367-374.

Wright, S. (1920). The relative importance of heredity and environment in determining the piebald pattern of guinea-pigs. Proceedings of the National Academy of Sciences, 6, 320-332.

Received September 6, 2001

Revision received July 31, 2002

Accepted October 23, 2002 